\title{
Dynamic consolidation problems in saturated soils solved through $u-w$ formulation in a LME meshfree framework
}

\author{
Pedro Navas ${ }^{a}$, Rena C. Yu ${ }^{a}$, Susana López-Querol ${ }^{b, 1}$, and Bo $\mathrm{Li}^{c}$ \\ ${ }^{a}$ E. T. S. de Ingenieros de Caminos, C. y P., Universidad de Castilla-La Mancha \\ 13071 Ciudad Real, Spain \\ ${ }^{b}$ Dept. of Civil, Environmental and Geomatic Engineering, University College London, \\ Gower Street, London WC1E 6BT, UK \\ ${ }^{c}$ Dept. of Mechanical and Aerospace Engineering, Case Western Reserve University, \\ Cleveland, Ohio 44106, USA
}

\begin{abstract}
A meshfree numerical model, based on the principle of Local Maximum Entropy (LME), including a B-bar algorithm to avoid instabilities, is applied to solve axisymmetric consolidation problems in elastic saturated soils. This numerical scheme has been previously validated for purely elastic problems without water (mono phase), as well as for steady seepage in elastic porous media. Hereinafter, an implementation of the novel numerical method in the axisymmetric configuration is proposed, and the model is validated for well known theoretical problems of consolidation in saturated soils, under both static and dynamic conditions with available analytical solutions. The solutions obtained with the new methodology are compared with a finite element commercial software for a set of examples. After validated, solutions for dynamic radial consolidation and sinks, which have not been found elsewhere in the literature, are presented as a novelty. This new numerical approach is demonstrated to be feasible for this kind of problems in porous media, particularly for high frequency, dynamic problems, for which very few results have been found in the literature in spite of their high practical importance.
\end{abstract}

Keywords: Meshfree, $u-w$ formulation, dynamic consolidation, B-bar.

\footnotetext{
${ }^{1}$ Corresponding author: S.Lopez-Querol@ucl.ac.uk
} 


\section{Introduction}

The consolidation of saturated media is a process in which the soil settles as a consequence of the application of external loading, causing a gradual interchange between pore pressure and effective stress after a certain period of time, which mainly depends on the permeability of the soil. Immediately after external loadings are applied to a saturated soil domain, all the external pressure transfers to water, certain amount of time being required for the dissipation of this excess pore water pressure to the solid phase. When this dissipation is complete (i.e total drainage has taken place), the solid phase totally takes the external pressure, which is converted to effective stress. This is what is meant by consolidation [1].

The external loads applied to the soil can be either static or variable in time, i.e. dynamic. In the former case, the evolution of water pressure displays a monotonic trend until the equilibrium is achieved, while in the latter, when dynamic external loadings are applied, the problem becomes further more complicated, because at the same time, generation and dissipation of water pressure take place, and coupling effects between solid and fluid phases need to be considered to achieve an accurate solution [2]. The frequency of the applied external loadings is a very important aspect to consider when a numerical strategy is to be selected in order to model the problem. It is widely recognised that the implementation of the Biot's equations [3] is a well-known way to solve problems in porous media from a macro-scale point of view. The advantage of this method is the possibility of accounting for coupling between phases. The $u-p_{w}$ formulation (where $u$ denotes the solid phase displacement, and $p_{w}$ is the pore fluid pressure) has been traditionally employed for simulating coupled problems in saturated porous media, although it has been demonstrated not to be a feasible approach when the frequency of the external loading is high [2]. The so-called complete or displacement based formulation, $u-w$ (where $w$ denotes the relative fluid displacement with respect to the solid phase) has been employed in several numerical schemes (López-Querol et al. [4], and recently adopted by Cividini and Gioda [5]). Such a methodology is employed in this work, first because of its simplicity in imposing impervious boundary conditions compared to the $u-p_{w}$ approaches; second, as the free surface comes out naturally as the zero-pressure contour, no detection algorithm is necessary; third, because its robustness makes it possible to model high frequency problems, in which the coupling between solid and fluid phase is more difficult to capture, and which are also very important from the practical point of view.

It is possible to find in the literature numerical models for consolidation problems which successfully account for real elastic-plastic soil behaviour 
$[6,7,8]$. Although meshfree numerical schemes have been known to perform particularly well in the regime of large deformations, in this paper only the small strain range is dealt with. Thus, we undertake such schemes to solve coupled problems in saturated porous media, using the $u-w$ formulation. The main aim of the present research is to explore the feasibility of the proposed B-bar based meshfree numerical tool to solve theoretical consolidation problems under the small strain range, particularly for high frequency problems.

The current work is a natural follow-up of the author's recent research on unconfined seepage flow through saturated soil [9] within a meshfree framework based on the principle of local maximum entropy [10]. In [9], a B-bar based algorithm was developed to avoid the volumetric locking problem encountered in displacement-based finite element approaches $[11,12,13,14$, $15,16,17,18]$ or meshfree approximation schemes [19]. The implementation takes advantage of the shape functions developed by Arroyo and Ortiz [20] and the OTM framework [21] for its numerous advantages in comparison with its alternatives. For example, the exact mass transport, the satisfaction of the continuity equation, exact linear and angular momentum conservation in order to solve different problems as spurious modes, tensile instabilities and unknown convergence or stability properties and convenient numerical integration scheme. Since the deformation and velocity fields are interpolated from nodal values using local max-ent shape functions, the Kronecker-delta property at the boundary makes it possible for the direct imposition of essential boundary conditions. In addition, the parameters pertinent to the local maximum entropy are obtained efficiently and and robustly, independently of the number of nodes in the support, through a combination of the Newton Raphson method and the Nelder Mead algorithm [22].

The rest of the paper is organised as follows: the mathematical framework, including the B-bar based algorithm, is presented next. After that, applications to various consolidation problems are illustrated in Section 4. Finally, the most relevant conclusions are drawn in Section 5.

\section{Mathematical framework}

In this section, we first summarise the governing equations for unconfined seepage problems, in particular the Biot's equations, formulated in a $u-w$ framework, which have been successfully utilised in $[5,9,23,24]$; next, the spacial discretisation based on the principle of maximum entropy is presented. 


\subsection{The Biot's equations: $a u-w$ formulation}

The Biot's equations [25] are based on formulating the mechanical behaviour of a solid-fluid mixture, the coupling between different phases, and the continuity of seepage through a differential domain of saturated porous media. In the following, $\boldsymbol{u}$ represents the displacement vector of the solid skeleton, whereas $\boldsymbol{w}$ denotes the relative displacement vector of the fluid phase with respect to the solid one. The advantages of the $u-w$ formulation when impermeability boundary conditions are imposed are well described in [23]: if there is no water displacement at those boundaries, the condition $\boldsymbol{w}=0$ can be easily established. This fact, in addition to the suitability of this method for dynamic problems, leads us to employ the complete formulation.

The final $u-w$ equations to solve are obtained by re-arranging the original Biot equations, as López-Querol explains in [26]:

$$
\begin{array}{r}
\boldsymbol{S}^{\boldsymbol{T}} \boldsymbol{D}^{e} \boldsymbol{S} d \boldsymbol{u}+Q \boldsymbol{\nabla}\left[\boldsymbol{\nabla}^{\boldsymbol{T}}(d \boldsymbol{u}+d \boldsymbol{w})\right]-\rho d \ddot{\boldsymbol{u}}-\rho_{f} d \ddot{\boldsymbol{w}}+\rho d \boldsymbol{b}=0 \\
Q \boldsymbol{\nabla}\left[\boldsymbol{\nabla}^{\boldsymbol{T}}(d \boldsymbol{u}+d \boldsymbol{w})\right]-\kappa^{-1} d \dot{\boldsymbol{w}}-\rho_{f} d \ddot{\boldsymbol{u}}-\frac{\rho_{f}}{n} d \ddot{\boldsymbol{w}}+\rho_{f} d \boldsymbol{b}=0
\end{array}
$$

where $\rho$ and $\rho_{f}$ are respectively the mixture and fluid phase densities, $\boldsymbol{b}$ is the external acceleration vector, $\kappa$ represents the permeability coefficient $\left(\kappa=k / \rho g\right.$, expressed in units $\left[\mathrm{m}^{3} \cdot \mathrm{s} / \mathrm{kg}\right.$, while $k$ is the hydraulic conductivity in $[\mathrm{m} / \mathrm{s}]) . \quad S$ is the differential operator and $Q$ is the volumetric compressibility of the mixture. Here $\boldsymbol{D}^{e}$ denotes the elastic stiffness tensor, assumed in this research as the plane strain constitutive tensor.

Equations (1) and (2) can be expressed as a system of equations, once the elementary matrices have been assembled:

$$
\boldsymbol{K} d \boldsymbol{u}+\boldsymbol{C} d \dot{\boldsymbol{u}}+\boldsymbol{M} d \ddot{\boldsymbol{u}}=d \boldsymbol{f}
$$

where $\boldsymbol{K}, \boldsymbol{C}$ and $\boldsymbol{M}$ respectively denote stiffness, damping and mass matrices, $d \boldsymbol{u}$ represents the vector of unknowns (containing both the solid phase and fluid displacements, $\boldsymbol{u}$ and $\boldsymbol{w}$ ), expressed incrementally, and $d \boldsymbol{f}$ is the increment of the vector of external forces, including gravity acceleration, as well as boundary conditions for nodal forces.

\subsection{Time discretisation}

A time integration algorithm is necessary to determine the solution of the problems dealt with in this paper. Even in the cases of static consolidation, in which the external loading is applied and kept constant in time, time integration is also required to capture the steady solution in terms of displacements and pressures when the consolidation process is nearly complete, and thus 
this numerical steady solution can be compared with analytical ones when they are available. For static problems, first order time integration schemes, neglecting the inertial terms, are usually sufficient, while for purely dynamic problems, second order of approximation is required most of the times to achieve stable and accurate enough solutions.

In this research, a standard first-order Newmark scheme has been employed for the static problems. Moreover, in order to be able to capture the effect of inertial terms in high frequency simulations, the Collocation time integration scheme has been considered more appropriate for the dynamic problems, some of them of high frequencies. This method was developed by Hughes and Hilber [27] as a Newmark and Wilson- $\theta$ [28] mix method. It introduces a numerical damping, allowing us to obtain a quick convergence in this kind of problems. This method converges to the Newmark solution for $\theta=1$, and to the Wilson- $\theta$ solution for $\alpha=1 / 6$ and $\delta=1 / 2$. Hughes and Hilber [27] demonstrated that the most stable form of this method is obtained using the following values of the parameters which control the stability of the algorithm:

$$
\delta=1 / 2 ; \theta \geq 1 ; \frac{\theta}{2(\theta+1)} \geq \alpha \geq \frac{2 \theta^{2}-1}{4\left(2 \theta^{2}-1\right)} .
$$

These restrictions for the parameters have been employed in the present work. In this study, $\theta$ and $\alpha$ have been respectively taken as 1.5 and 0.273 .

As in all step-by-step time integration schemes, it is necessary to divide the time domain into steps, with time interval, $\Delta t$, small enough to warrant both convergence and accuracy of the solution. In this paper, most of the problems consist of the application of harmonic loads. The maximum $\Delta t$ needs to be taken as $T / 10$, where $T$ is the period of the external load.

Rearranging the above expressions, Eq. (3) finally yields

$$
\begin{gathered}
{\left[\frac{1}{\alpha \Delta t^{2} \theta^{2}} \boldsymbol{M}+\frac{1}{\alpha \Delta t \theta} \boldsymbol{C}+\boldsymbol{K}\right] \Delta \boldsymbol{u}_{\theta \Delta t}=\Delta \boldsymbol{f}_{\Delta t \theta}+\Delta \boldsymbol{R}_{n}+} \\
\boldsymbol{C}\left[\frac{\delta}{\alpha} \dot{\boldsymbol{u}}_{n}-\left(1-\frac{\delta}{2 \alpha}\right) \Delta t \theta \ddot{\boldsymbol{u}}_{n}\right]+\boldsymbol{M}\left[\frac{1}{\alpha \Delta t \theta} \dot{\boldsymbol{u}}_{n}+\frac{1}{2 \alpha} \ddot{\boldsymbol{u}}_{n}\right]
\end{gathered}
$$

where

$$
\Delta \boldsymbol{R}_{n}=\boldsymbol{f}_{n}-\boldsymbol{M} \ddot{\boldsymbol{u}}_{n}-\boldsymbol{C} \dot{\boldsymbol{u}}_{n}-\boldsymbol{K} \boldsymbol{u}_{n} .
$$

\subsection{Spatial discretisation: Max-ent shape functions}

The local max-ent approximation scheme is defined by Arroyo and Ortiz [20], and employed by $\mathrm{Li}$ et al. [21] for fields requiring differentiation, such as deformation and velocity fields. Arroyo and Ortiz [20] defined the local 
max-ent function (LME) as a Pareto set, being optimal for $\beta \in(0, \infty)$. The shape function is obtained as:

$$
p_{a}(\mathbf{x})=\frac{\exp \left[-\beta\left|\mathbf{x}-\mathbf{x}_{\mathbf{a}}\right|^{2}+\boldsymbol{\lambda}^{*} \cdot\left(\mathbf{x}-\mathbf{x}_{\mathbf{a}}\right)\right]}{Z\left(\mathbf{x}, \boldsymbol{\lambda}^{*}(\mathbf{x})\right)},
$$

where

$$
Z(\mathbf{x}, \boldsymbol{\lambda})=\sum_{a=1}^{n} \exp \left[-\beta\left|\mathbf{x}-\mathbf{x}_{\mathbf{a}}\right|^{2}+\boldsymbol{\lambda} \cdot\left(\mathbf{x}-\mathbf{x}_{\mathbf{a}}\right)\right],
$$

being $\boldsymbol{\lambda}^{*}(\mathbf{x})$ the unique minimiser for $\log Z(\mathbf{x}, \boldsymbol{\lambda})$. The parameter $\beta$ is related with the discretisation size (or nodal spacing), $h$, and the constant, $\gamma$, which controls the locality of the shape functions, as follows,

$$
\beta=\frac{\gamma}{h^{2}}
$$

For a uniform nodal spacing, $\beta$ is also a constant, thus the first derivatives can be obtained by employing Arroyo and Ortiz [20] research with the following expression:

$$
\nabla p_{a}^{*}=-p_{a}^{*}\left(\mathbf{J}^{*}\right)^{-1}\left(\mathbf{x}-\mathbf{x}_{\mathbf{a}}\right),
$$

where $\mathbf{J}$ is the Hessian matrix, defined by:

$$
\begin{aligned}
\mathbf{J}(\mathbf{x}, \boldsymbol{\lambda}, \beta) & =\frac{\partial \mathbf{r}}{\partial \boldsymbol{\lambda}} \\
\mathbf{r}(\mathbf{x}, \boldsymbol{\lambda}, \beta) & \equiv \partial_{\boldsymbol{\lambda}} \log Z(\mathbf{x}, \boldsymbol{\lambda})=\sum_{a} p_{a}(\mathbf{x}, \boldsymbol{\lambda}, \beta)\left(\mathbf{x}-\mathbf{x}_{\mathbf{a}}\right)
\end{aligned}
$$

Note that, the objective of the above procedure is to find the $\boldsymbol{\lambda}$ which minimises $\log Z(\mathbf{x}, \boldsymbol{\lambda})$. This unconstrained minimization problem with a strictly convex objective function can be solved efficiently and robustly by a combination of the Newton-Raphson method and Nelder-Mead Simplex algorithm $[9,20,22]$.

\section{B-bar based algorithm: extension to axisymmetric formulation}

The B-bar algorithm developed by the authors in [9] is based on the strain projection method, which is typically characterised by an interpolation of the discrete gradient operator a-priori assumed, independently of the approximation adopted for the displacement. In particular, the proposed assumed strain field was developed by averaging the volumetric strain among a cluster of material points defined in the OTM framework, which falls within 
the class of often referred to B-bar procedure introduced by Hughes [11]. The introduction of a material point discretisation and the local maximum entropy (LME) meshfree approximating subspace within the OTM framework yields no influence on the construction of a general B-bar procedure proposed by Simo and Hughes [29]. For completeness, we summarise it here to facilitate its extension to axisymmetric coordinate systems.

The strain tensor $\varepsilon\left(\theta_{p}\right)$ computed at a material point $\theta_{p}$ is transformed to $\bar{\varepsilon}\left(\theta_{p}\right)$ by replacing its volumetric part to an averaged one evaluated on a cluster of material points, or the patch associated with the material point, i.e.,

$$
\bar{\varepsilon}\left(\theta_{p}\right)=\varepsilon^{\operatorname{dev}}\left(\theta_{p}\right)+\pi\left(\varepsilon^{\mathrm{vol}}\left(\theta_{p}\right)\right)
$$

and the projection function, $\pi$, is defined as

$$
\pi\left(\varepsilon^{\mathrm{vol}}\left(\theta_{p}\right)\right)=\overline{\varepsilon^{\mathrm{vol}}\left(\theta_{p}\right)}=\frac{\sum_{q \in \mathcal{I}\left(\Omega_{p}\right)} \varepsilon^{\mathrm{vol}}\left(\theta_{q}\right) v^{(q)}}{\sum_{q \in \mathcal{I}\left(\Omega_{p}\right)} v^{(q)}}=\sum_{q \in \mathcal{I}\left(\Omega_{p}\right)} \varepsilon^{\mathrm{vol}}\left(\theta_{q}\right) w^{(q)}
$$

where $\Omega_{p}$ is the patch associated with material point $\theta_{p}$ or the cluster of material points which contains $\theta_{p}, v^{(q)}$ is the volume of material point $\theta_{q}$ belonging to $\Omega_{p}$, and $\mathcal{I}\left(\Omega_{p}\right)$ is the index set of the material points in $\Omega_{p}$ and $w^{(q)}$ is a weight obtained from the volume:

$$
w^{(q)}=\frac{v^{(q)}}{\sum_{q \in \mathcal{I}\left(\Omega_{p}\right)} v^{(q)}} .
$$

The deviatoric and volumetric part of the strain tensor are defined as

$$
\varepsilon^{\mathrm{vol}}\left(\theta_{p}\right)=\frac{1}{d} \operatorname{tr}\left(\varepsilon\left(\theta_{p}\right)\right) \boldsymbol{I} \quad \text { and } \quad \varepsilon^{\operatorname{dev}}\left(\theta_{p}\right)=\varepsilon\left(\theta_{p}\right)-\varepsilon^{\mathrm{vol}}\left(\theta_{p}\right),
$$

respectively, where $d$ is the dimensional of the problem.

Next we explain in detail the procedure to calculate the B-bar matrix for an axisymmetric framework.

\subsection{B-bar implementation in $u-w$ axisymmetric problems}

Applications of the LME meshfree approximation in an axisymmetric soil consolidation problem using $u-w$ framework have not been investigated in the literature. In this work, we re-visit the formulation proposed in [9] for the LME interpolation of the $u, w$ fields and the B-bar algorithm in the axisymmetric coordinate system. In axisymmetric problems, the radial and vertical directions ( $r$ and $z$ ) play the role of $x$ and $y$ in a 2D, cartesian case. Consequently, the new displacement vector is related to the nodal vectors 
through the shape function based on the principle of local maximum entropy (LME) in a similar way:

$$
\left[\begin{array}{c}
u_{r} \\
u_{z} \\
w_{r} \\
w_{z}
\end{array}\right]=\left[\begin{array}{ccccccccc}
N_{1} & 0 & 0 & 0 & N_{2} & 0 & 0 & 0 & \ldots \\
0 & N_{1} & 0 & 0 & 0 & N_{2} & 0 & 0 & \ldots \\
0 & 0 & N_{1} & 0 & 0 & 0 & N_{2} & 0 & \ldots \\
0 & 0 & 0 & N_{1} & 0 & 0 & 0 & N_{2} & \ldots
\end{array}\right]\left[\begin{array}{c}
u_{r 1}^{h} \\
u_{z 1}^{h} \\
w_{r 1}^{h} \\
w_{z 1}^{h} \\
u_{r 2}^{h} \\
u_{z 2}^{h} \\
w_{r 2}^{h} \\
w_{z 2}^{h} \\
\vdots
\end{array}\right]
$$

where the superscript ${ }^{h}$ denotes discrete nodal values. In this Section, we carry out the same procedure as a $2 \mathrm{D}$ multiphase problem by splitting the strain tensor into its solid and fluid components, we have

$$
\boldsymbol{\varepsilon}=\boldsymbol{S} \boldsymbol{u} \longrightarrow\left[\begin{array}{c}
\boldsymbol{\varepsilon}^{s} \\
\boldsymbol{\varepsilon}^{\boldsymbol{w}}
\end{array}\right]=\left[\begin{array}{c}
\varepsilon_{r}^{s} \\
\varepsilon_{z}^{s} \\
\varepsilon_{\theta}^{s} \\
\gamma_{r z}^{s} \\
\varepsilon_{r}^{w} \\
\varepsilon_{z}^{w} \\
\varepsilon_{\theta}^{w}
\end{array}\right]=\left[\begin{array}{cccc}
\frac{\partial}{\partial r} & 0 & 0 & 0 \\
0 & \frac{\partial}{\partial z} & 0 & 0 \\
\frac{1}{r} & 0 & 0 & 0 \\
\frac{\partial}{\partial z} & \frac{\partial}{\partial r} & 0 & 0 \\
0 & 0 & \frac{\partial}{\partial r} & 0 \\
0 & 0 & 0 & \frac{\partial}{\partial z} \\
0 & 0 & \frac{1}{r} & 0
\end{array}\right]\left[\begin{array}{c}
u_{r} \\
u_{z} \\
w_{r} \\
w_{z}
\end{array}\right],
$$

where, the superscripts ${ }^{s}$ and ${ }^{w}$ denote the solid and fluid phases respectively. In addition, the sum of the strain traces of the solid and fluid phases can be done through the unit matrix, $\boldsymbol{m}^{*}$, in Voigt notation as follows:

$$
\operatorname{tr}\left(\boldsymbol{\varepsilon}^{\boldsymbol{s}}\right)+\operatorname{tr}\left(\boldsymbol{\varepsilon}^{\boldsymbol{w}}\right)=\left(\boldsymbol{m}^{*}\right)^{T} \boldsymbol{\varepsilon}
$$

where:

$$
\left(\boldsymbol{m}^{*}\right)^{T}=\left[\begin{array}{lllllll}
1 & 1 & 1 & 0 & 1 & 1 & 1
\end{array}\right]
$$

148 Hence, the new constitutive matrix which relates $\boldsymbol{\sigma}$ and $\varepsilon$ yields:

$$
\begin{aligned}
\boldsymbol{\sigma} & =\boldsymbol{D}^{e *} \boldsymbol{\varepsilon}+Q \boldsymbol{m}^{T} \boldsymbol{\varepsilon} \boldsymbol{m}=\left(\boldsymbol{D}^{e *}+Q \boldsymbol{m}^{T} \boldsymbol{m}\right) \boldsymbol{\varepsilon}=\boldsymbol{D}^{\boldsymbol{u}-\boldsymbol{w}} \boldsymbol{\varepsilon} \\
= & {\left[\begin{array}{ccccccc}
\frac{\lambda(1-\nu)}{\nu}+Q & \lambda+Q & \lambda+Q & 0 & Q & Q & Q \\
\lambda+Q & \frac{\lambda(1-\nu)}{\nu}+Q & \lambda+Q & 0 & Q & Q & Q \\
\lambda+Q & \lambda+Q & \frac{\lambda(1-\nu)}{\nu}+Q & 0 & Q & Q & Q \\
0 & 0 & 0 & \mu & 0 & 0 & 0 \\
Q & Q & Q & 0 & Q & Q & Q \\
Q & Q & Q & 0 & Q & Q & Q \\
Q & Q & Q & 0 & Q & Q & Q
\end{array}\right]\left[\begin{array}{c}
\varepsilon_{r}^{s} \\
\varepsilon_{z}^{s} \\
\varepsilon_{\theta}^{s} \\
\gamma_{r z}^{s} \\
\varepsilon_{r}^{w} \\
\varepsilon_{z}^{w} \\
\varepsilon_{\theta}^{w}
\end{array}\right] }
\end{aligned}
$$


For the purpose of implementing the B-Bar based algorithm, the strain tensor can be re-calculated as $\varepsilon$. Thus, the main equation yields:

$$
\overline{\boldsymbol{\varepsilon}}=\boldsymbol{\varepsilon}-\frac{1}{d} \operatorname{tr}\left(\boldsymbol{\varepsilon}^{\boldsymbol{s}}\right) \mathbf{I}+\frac{1}{d}\left[\operatorname{tr}\left(\boldsymbol{\varepsilon}^{\boldsymbol{s}}\right)\right]^{p} \mathbf{I}-\frac{1}{d} \operatorname{tr}\left(\boldsymbol{\varepsilon}^{\boldsymbol{w}}\right) \mathbf{I}+\frac{1}{d}\left[\operatorname{tr}\left(\boldsymbol{\varepsilon}^{\boldsymbol{w}}\right)\right]^{p} \mathbf{I} .
$$

In Voigt notation the equation, the corresponding $l^{\text {th }}$-component is:

$$
\overline{\varepsilon_{l}}=\varepsilon_{l}+\frac{1}{d}\left(-\varepsilon_{k k} m_{l}^{s}+\sum_{j=1}^{N_{b}}\left[\varepsilon_{k k}^{(j)} w^{(j)}\right] m_{l}^{s}-\varepsilon_{k k}^{w} m_{l}^{w}+\sum_{j=1}^{N_{b}}\left[\varepsilon_{k k}^{w(j)} w^{(j)}\right] m_{l}^{w}\right)
$$

where the solid and fluid parts are related with the global strain through the following expressions:

$$
\varepsilon_{k k}^{s}=m_{k}^{s} \varepsilon_{k}, \quad \varepsilon_{k k}^{w}=m_{k}^{w} \varepsilon_{k} .
$$

The unit matrix in Voigt notation for the solid and fluid case are as follows

$$
\left(m^{s}\right)^{T}=\left[\begin{array}{lllllll}
1 & 1 & 1 & 0 & 0 & 0 & 0
\end{array}\right], \quad\left(m^{w}\right)^{T}=\left[\begin{array}{lllllll}
0 & 0 & 0 & 0 & 1 & 1 & 1
\end{array}\right] .
$$

Alternatively, we know that the $l$-th component of the strain tensor in Voigt notation is:

$$
\varepsilon_{l}=S_{l j} u_{j}=S_{l j} N_{j k} u_{k}^{h}=B_{l k} u_{k}^{h},
$$

where, in this case, yields:

$$
\left[\begin{array}{c}
\varepsilon_{r} \\
\varepsilon_{z} \\
\varepsilon_{\theta} \\
\gamma_{r z} \\
\varepsilon_{r}^{w} \\
\varepsilon_{z}^{w} \\
\varepsilon_{\theta}^{w}
\end{array}\right]=\left[\begin{array}{cccc|cccc|c}
\frac{\partial N_{1}}{\partial r} & 0 & 0 & 0 & \frac{\partial N_{2}}{\partial r} & 0 & 0 & 0 \\
0 & \frac{\partial N_{1}}{\partial z} & 0 & 0 & 0 & \frac{\partial N_{2}}{\partial z} & 0 & 0 \\
\frac{N_{1}}{r} & 0 & 0 & 0 & \frac{N_{2}}{r} & 0 & 0 & 0 \\
\frac{\partial N_{1}}{\partial z} & \frac{\partial N_{1}}{\partial r} & 0 & 0 & \frac{\partial N_{2}}{\partial z} & \frac{\partial N_{2}}{\partial r} & 0 & 0 \\
0 & 0 & \frac{\partial N_{1}}{\partial r} & 0 & 0 & 0 & \frac{\partial N_{2}}{\partial r} & 0 \\
0 & 0 & 0 & \frac{\partial N 1}{\partial z} & 0 & 0 & 0 & \frac{\partial N_{2}}{\partial z} \\
0 & 0 & \frac{N_{1}}{r} & 0 & 0 & 0 & \frac{N_{2}}{r} & 0
\end{array}\right] \cdots\left[\begin{array}{c}
u_{r}^{(1)} \\
u_{z}^{(1)} \\
w_{r}^{(1)} \\
w_{z}^{(1)} \\
u_{r}^{(2)} \\
u_{z}^{(2)} \\
w_{r}^{(2)} \\
w_{z}^{(2)} \\
\vdots
\end{array}\right]
$$

In order to calculate the strain trace, $\varepsilon_{l l}$, by re-arranging different terms, we can obtain for the solid phase:

$$
\varepsilon_{l l}^{s}=m_{l}^{s} \varepsilon_{l}=m_{l} B_{l k} u_{k}^{h}=T_{k}^{s} u_{k}^{h},
$$

where

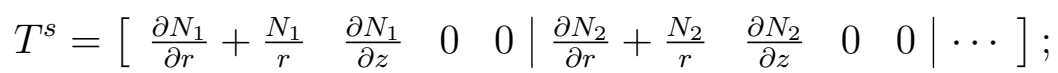


for the fluid phase

$$
\varepsilon_{l l}^{w}=m_{l}^{w} \varepsilon_{l}=m_{l} B_{l k} u_{k}^{h}=T_{k}^{w} u_{k}^{h},
$$

where

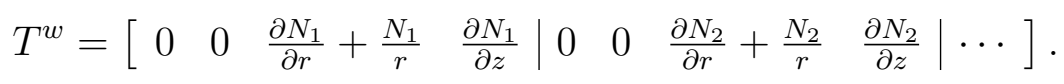

Thus, the final $l^{t h}$-component for the new strain tensor $\bar{\varepsilon}$ at a single integration point $i$ in Voigt notation is calculated as:

$$
\begin{aligned}
\bar{\varepsilon}_{l}^{(i)}=B_{l k}^{(i)} u_{k}^{h} & -\frac{1}{d} m_{l}^{s}\left(T_{k}^{s(i)} u_{k}^{h}-\sum_{j=1}^{N_{b}}\left[T_{k}^{s(j)} w^{(j)}\right] u_{k}^{h}\right) \\
& -\frac{1}{d} m_{l}^{w}\left(T_{k}^{w(i)} u_{k}^{h}-\sum_{j=1}^{N_{b}}\left[T_{k}^{w(j)} w^{(j)}\right] u_{k}^{h}\right) \\
= & {\left[B_{l k}^{(i)}-\frac{1}{d} m_{l}^{s}\left(T_{k}^{s(i)}-\sum_{j=1}^{N_{b}}\left[T_{k}^{s(j)} w^{(j)}\right]\right)\right.} \\
& \left.-\frac{1}{d} m_{i}^{w}\left(T_{k}^{w(i)}-\sum_{j=1}^{N_{b}}\left[T_{k}^{w(j)} w^{(j)}\right]\right)\right] u_{k}^{h} \\
\equiv & \overline{B_{l k}} u_{k}^{h} .
\end{aligned}
$$

\section{Application to consolidation in soils}

As previously mentioned, the settlement of saturated soils under loading is caused by a gradual interchange between pore pressure and effective stress. This process is known as consolidation. In this section, the above developed methodology is applied to consolidation in soils in three different configurations: one dimensional case (for validation purposes), radial consolidation, and consolidation with sinks. Both static and dynamic scenarios, with different frequencies ranging from low to high values, are studied. The obtained solutions are compared with analytical or available numerical solutions.

\subsection{Consolidation of a soil column: the one-dimensional static problem}

One-dimensional consolidation in a soil column under vertical loading occurs when there is no lateral strain, and only vertical displacements of both solid and fluid phases are developed. Analytical solutions for this problem are derived from the basic equation given by Terzaghi in 1925 [30]. 


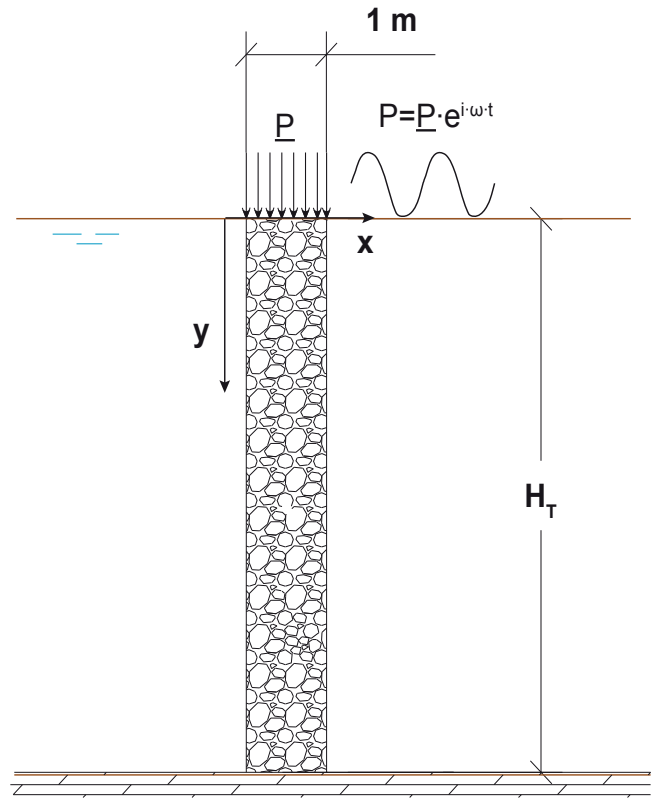

Base layer, impermeable
- Nodes

- Material Points

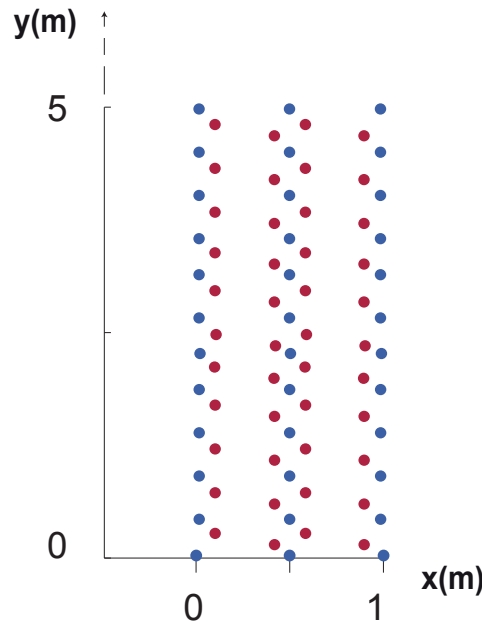

Figure 1: Geometry, loading condition of the consolidation column, and the discretised nodes and material points (shown for the first $5 \mathrm{~m}$ only). The same geometry has been employed for both static and dynamic simulations.

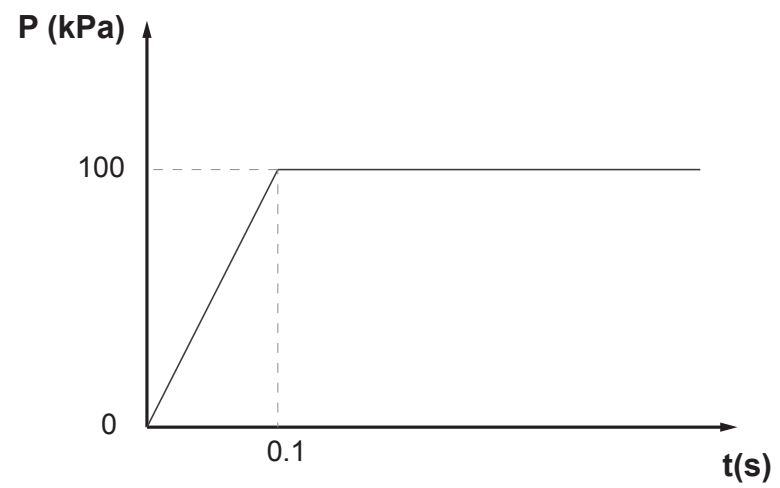

Figure 2: Loading history in a monotonic problem. 


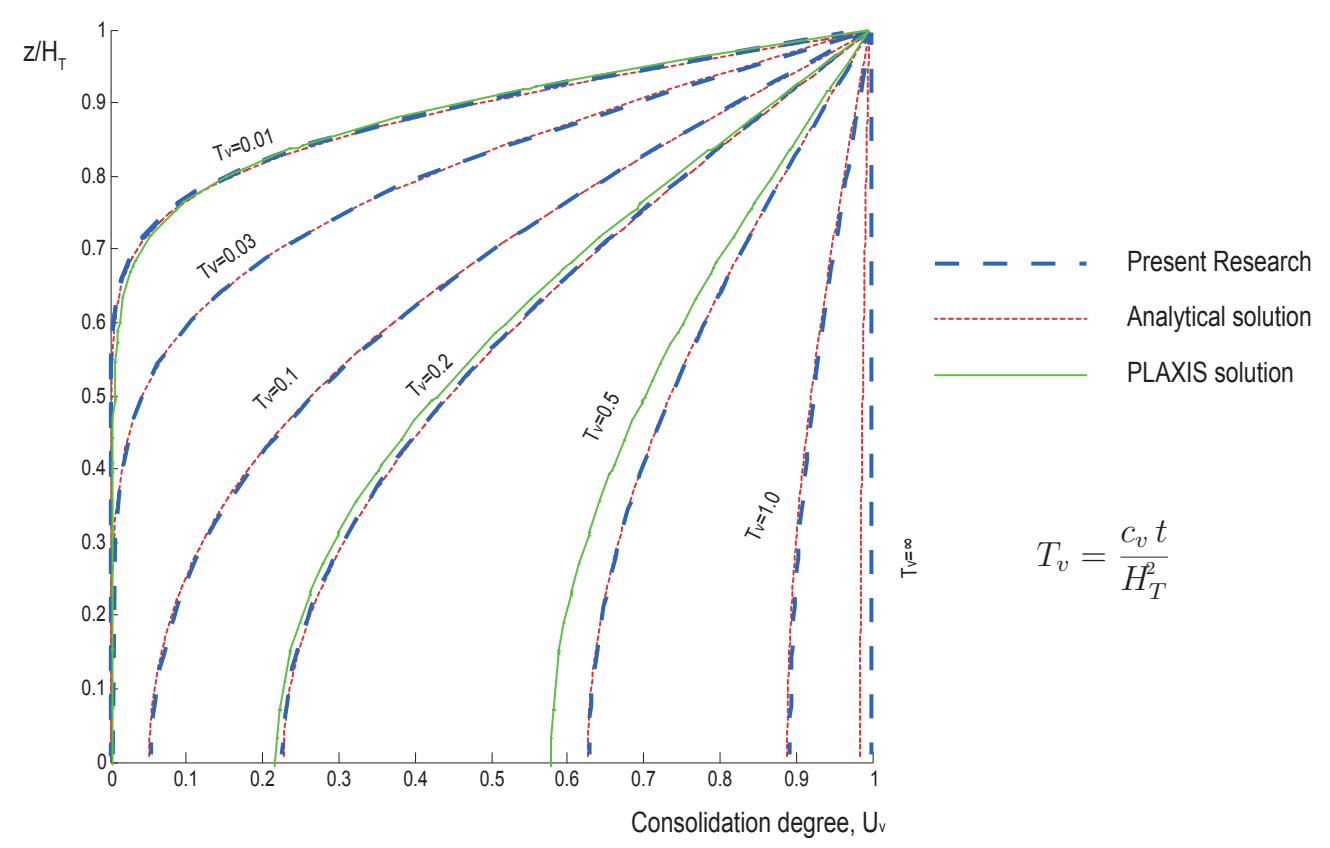

Figure 3: Comparison of analytical and numerical solutions of degree of consolidation, $U_{v}(z)$ at different values of dimensionless time, $T_{v}$.

In order to validate our numerical model against the analytical solution given in [31], a 30-metre-deep elastic soil column, depicted in Fig. 1, is modelled. The column rests over an impermeable rigid base layer and loaded by a vertical, uniform pressure on the top. The lateral displacements are restricted for both solid and fluid phases. At the base layer, the vertical displacements for both solid and fluid phases are prevented. Thus the onedimension draining condition is ensured. The column is discretised into 240 nodes and 183 material points. In this case, the external loading is static, but is gradually applied as depicted in Fig. 2. The consolidation behaviour is led by the vertical consolidation coefficient, $c_{v}$. As for elastic parameters, typical values for clays have been adopted: $2 \mathrm{MPa}$ for the Young's modulus and 0.33 for the Poisson's ratio.

In Fig. 3, analytical and numerical solutions along the depth of the column of soil for different values of the dimensionless time, $T_{v}$, are compared in a non-dimensional way. Note that the solution given by this research coincides with the analytical one except when $T_{v}$ approaches infinity. In comparison with the one obtained with the commercial software PLAXIS in Fig. 3, for lower values of $T_{v}$, the solution of the degree of consolidation, $U_{v}$, given by 


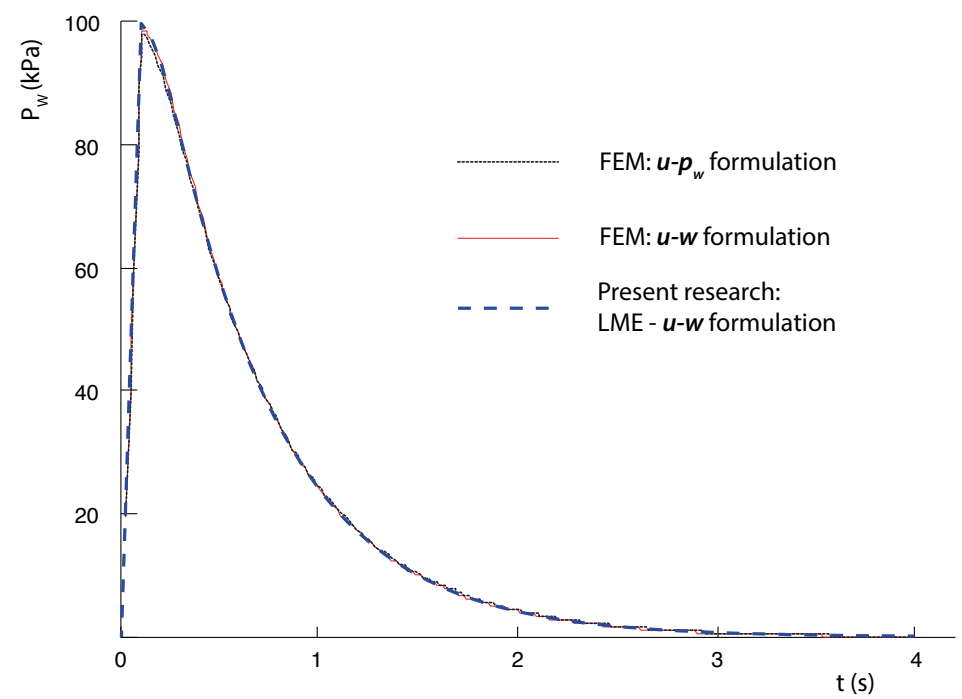

Figure 4: Comparison of different pore pressure evolution solutions at the top of the consolidation column [26, 32].

the commercial program along the column of soil is similar to that of the current work, although significant discrepancy is observed for higher values of $T_{v}$, while our proposed solution is still close to the analytical one. With this example, we have validated the numerical method for static consolidation problems.

In Fig. 4, the dissipation of excess pore pressure over time at the top of the soil column under the monotonic loading, as given in Fig. 2, is obtained using the current methodology, as well as with quadratic finite element codes under $u-w$ or $u-p_{w}$ formulations $[26,32]$. Note that the difference is hardly detectable.

In Fig. 5, the comparison is shown for the isochrone at time $0.1 \mathrm{~s}$ for a soil permeability of $0.167 \times 10^{-4} \mathrm{~m} / \mathrm{s}$. Note that when the impervious layer is approached, instability is observed for the solution obtained with $u-$ $p_{w}$ formulation. Such an instability is overcome with the current meshfree methodology by utilizing a finer discretisation and tuning the parameter $\gamma$ in Eq. (9). Since the $\gamma$-parameter controls the influence radius of the LME shape functions and a smaller $\gamma$ alleviates the problem of volume locking as there are more neighbour nodes contributing to the value of the pore pressure of the material point, more details can be found in the author's recent work [9]. The advantages of the $u-w$ formulation over the conventional $u-p_{w}$ are evidenced, in particular, for such dynamic problems. 


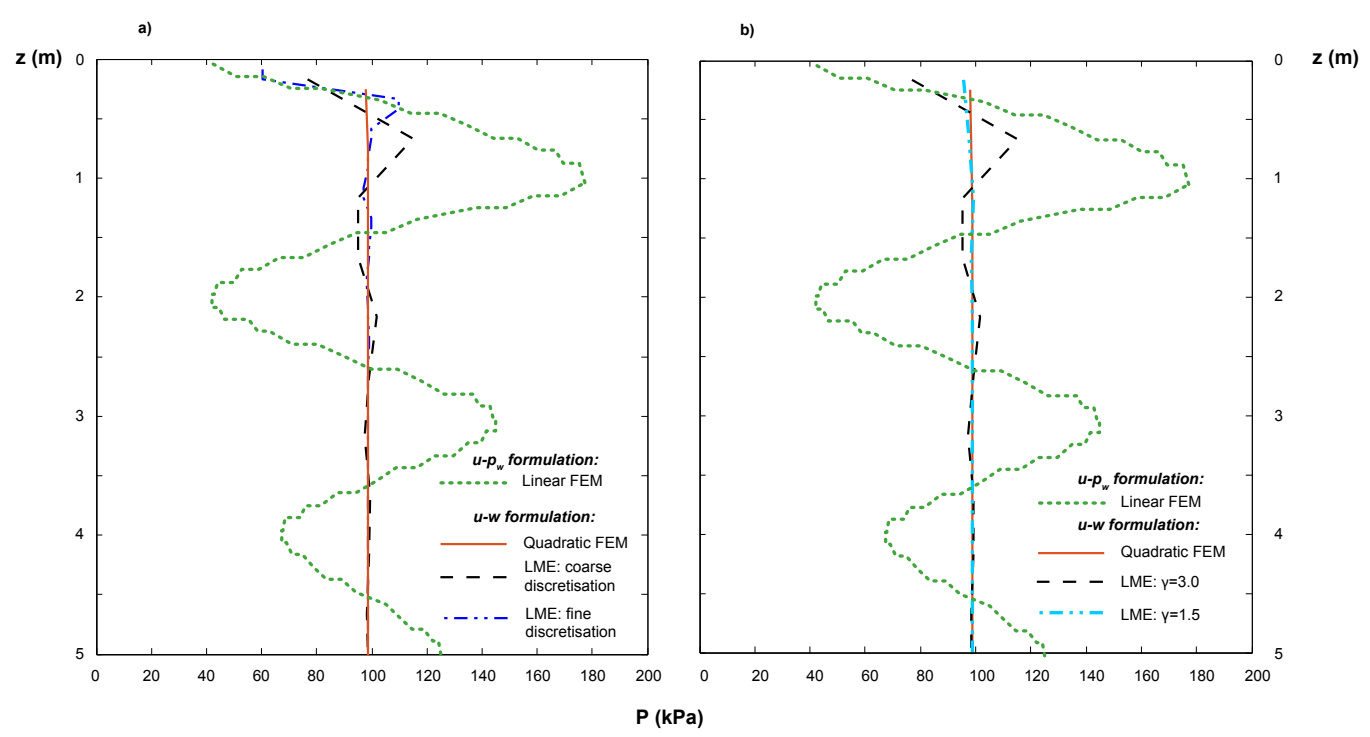

Figure 5: Comparison of the isochrones at $t=0.1 \mathrm{~s}$ obtained using $u-w$ and $u-p_{w}$ formulations in finite-element and current meshfree solutions with a) different levels of discretisation b) different $\beta$ values [26, 32].

\subsection{Consolidation of a soil column: the dynamic problem in $1 D$}

In this Section, the dynamic consolidation of a soil column is studied using the same geometry given in Fig. 1. In this case, the external loading is replaced by a harmonic pressure at the top. This problem was first analytically solved by Zienkiewicz et al. [2] in 1980s, and more recently by López-Querol [26] using a quadratic finite element method. The material parameters are provided in Table 1, and they are chosen to fit the dimensionless parameters employed by Zienkiewicz et al. [2]. For example, the density $\rho$ in Table 1, does not correspond to a real soil, but is chosen to obtain the same $\rho_{f} / \rho$ ratio as that of given in [2]. The amplitude of the loading is $100 \mathrm{kPa}$, while the frequency ranges from low to high values, to cover all the possible types of dynamic consolidation problems defined in [2]. The variation of the pore pressure with depth is calculated for different values of the dimensionless parameters $\Pi_{1}$ and $\Pi_{2}$, which are defined as follows [2]:

$$
\Pi_{1}=\frac{k V_{c}^{2}}{g \frac{\rho_{f}}{\rho} \omega H_{T}^{2}}=\frac{k \omega}{g \frac{\rho_{f}}{\rho} \Pi_{2}}, \quad \Pi_{2}=\frac{\omega^{2} H_{T}^{2}}{V_{c}^{2}}
$$

where $H_{T}$ is the column height, $V_{c}$ is the $p$-wave velocity calculated as:

$$
V_{c}=\sqrt{\left(D+\frac{K_{f}}{n}\right) \frac{1}{\rho}},
$$


a)

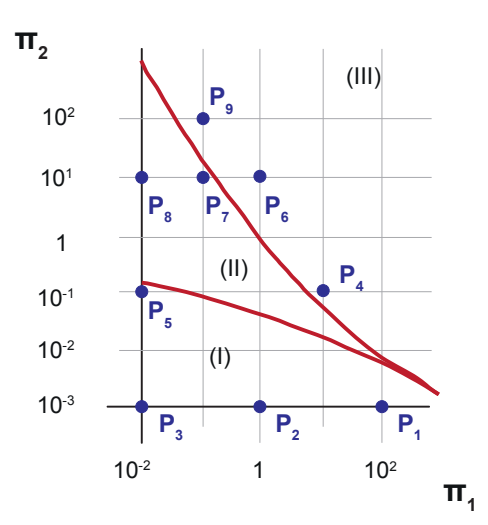

b)

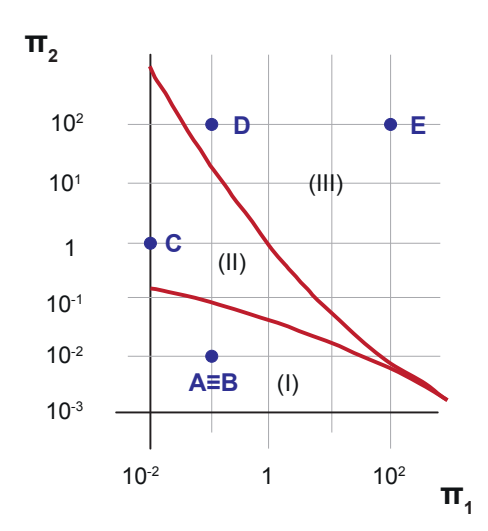

Zone (I) - Slow phenomena: ü and $\ddot{w}$ can be neglected

Zone (II) - Moderate speed: W can be neglected

Zone (III) - Fast phenomena: only full Biot eq. valid

Figure 6: Zones of the different behaviour of the soil depending on the parameters $\Pi_{1}$ and $\Pi_{2}[2]$ showing simulations carried out in a) $1 \mathrm{D}$ column problem; and b) radial, sink and Lamb's consolidation problem.

In the above expression, $D$ stands for the constrained modulus of the soil. Note that, since $\omega /(2 \pi)$ is the frequency of forced motion (external load), whereas $V_{c} /\left(2 H_{T}\right)$ is the representative natural frequency of the system (the soil column), the parameter $\Pi_{2}$ is closely related with ratio between the two. The parameter $\Pi_{1}$ combines this ratio together with the influence of the hydraulic conductivity, the loading frequency and the relative density between the fluid and the dry mixture.

Different soil behaviours can be distinguished according to the values of $\Pi_{1}$ and $\Pi_{2}$. The three different zones classified in this manner by Zienkiewicz et al. [2] are illustrated in Fig. 6. Zone I is characterised as slow phenomenon where both solid and fluid accelerations can be neglected; Zone II is typical of moderate speed behaviour, where only the fluid phase inertia is negligible; in Zone III, however, inertial contributions from both solid and fluid phases are significant and cannot be neglected.

Table 1: Material parameters employed for the dynamic consolidation problem of a soil column (where $G$ is the shear modulus).

\begin{tabular}{ccccc|cc|c}
\hline $\begin{array}{c}\mathrm{G} \\
{[\mathrm{MPa}]}\end{array}$ & $\begin{array}{c}\nu \\
-\end{array}$ & $\begin{array}{c}\mathrm{n} \\
{\left[\mathrm{kg} / \mathrm{m}^{3}\right]}\end{array}$ & $\begin{array}{c}\rho_{f} \\
{\left[\mathrm{~kg} / \mathrm{m}^{3}\right]}\end{array}$ & $\begin{array}{c}K_{f} \\
{[\mathrm{MPa}]}\end{array}$ & $\begin{array}{c}K_{s} \\
{[\mathrm{MPa}]}\end{array}$ & $\begin{array}{c}V_{c} \\
{[\mathrm{~m} / \mathrm{s}]}\end{array}$ \\
\hline 312.5 & 0.2 & 0.333 & 3003 & 1000 & $10^{4}$ & $10^{34}$ & 3205 \\
\hline
\end{tabular}


Note that for the given material properties in Table $1, \Pi_{2}$ is directly related to the angular velocity of loading, $w$, whereas $\Pi_{1}$ is also influenced by the the hydraulic conductivity, $k$. The parameter values to define the nine points, from $\mathrm{P}_{1}$ to $\mathrm{P}_{9}$, depicted in Fig. 6a), are listed in Table 2. For given $k$ and $\omega$, transient calculations are performed to obtain the maximum envelop of the pore pressure history for different points along the column depth and the excess pore water pressure distribution at a give time (i.e. isochrones). These results are then compared with the analytical solution given by Zienkiewicz et al. [2] in Figs. 7-10 to check the accuracy of the current methodology.

Table 2: The parameters, $k$ and $\omega$, for $H_{T}=10 \mathrm{~m}$ and different $\Pi_{1}$ and $\Pi_{2}$ values for $\mathrm{P}_{1}$ to $\mathrm{P}_{9}$ in Fig.7a).

\begin{tabular}{l|clll}
\hline$\Pi_{2}$ & $10^{-3}$ & $10^{-1}$ & $10^{1}$ & $10^{2}$ \\
\hline$\omega[\mathrm{rad} / \mathrm{s}]$ & 10.14 & 101.4 & 1014 & 3206 \\
\hline$\Pi_{1}$ & \multicolumn{5}{|l}{$k[\mathrm{~m} / \mathrm{s}]$} \\
\hline \hline $10^{2}$ & $3.22 \mathrm{E}-2\left(\mathrm{P}_{1}\right)$ & $3.22 \mathrm{E}-2\left(\mathrm{P}_{4}\right)$ & \\
$10^{1}$ & & $3.22 \mathrm{E}-2\left(\mathrm{P}_{6}\right)$ & \\
$10^{0}$ & $3.22 \mathrm{E}-4\left(\mathrm{P}_{2}\right)$ & & $3.22 \mathrm{E}-3\left(\mathrm{P}_{7}\right)$ & $1.018 \mathrm{E}-2\left(\mathrm{P}_{9}\right)$ \\
$10^{-1}$ & & & \\
$10^{-2}$ & $3.22 \mathrm{E}-6\left(\mathrm{P}_{3}\right)$ & $3.22 \mathrm{E}-5\left(\mathrm{P}_{5}\right)$ & $3.22 \mathrm{E}-4\left(\mathrm{P}_{8}\right)$ & \\
\hline
\end{tabular}

For the three points $\mathrm{P}_{1}, \mathrm{P}_{2}$ and $\mathrm{P}_{3}$ located in Zone $\mathrm{I}, \Pi_{2}$ is kept constant, which means the external loads are of the same frequency. The parameter $\Pi_{1}$ covers four orders of magnitude, so does the soil permeability. The isochrones of the pore pressure are compared with the analytical ones in Fig. 7. It needs to be pointed out that Zienkiewicz's solution is calculated neglecting the accelerations, whereas the present research solution is a second-order approximation taking into consideration the inertia term. No significant differences between both approaches are appreciated. This confirms that, as indicated by Zienkiewicz et al. [2], in Zone I, the inertial terms can be ignored. The solution obtained using a finite element code is also included, demonstrating the similarity with the solutions derived with the methodology presented in this paper.

Employing the different values of $k$ and $\omega$ listed in Table 2, the obtained isochrones for points $\mathrm{P}_{4}$ to $\mathrm{P}_{9}$ are compared with the analytical ones given by Zienkiewicz et al. [2] as well as the solution obtained with FEM, employing $u-w$ formulation for $\Pi_{2}$ from $10^{-1}$ to $10^{2}$ in Figs. 8-10. The higher is the parameter $\Pi_{2}$, the more unstable is the problem. In spite of that, the 


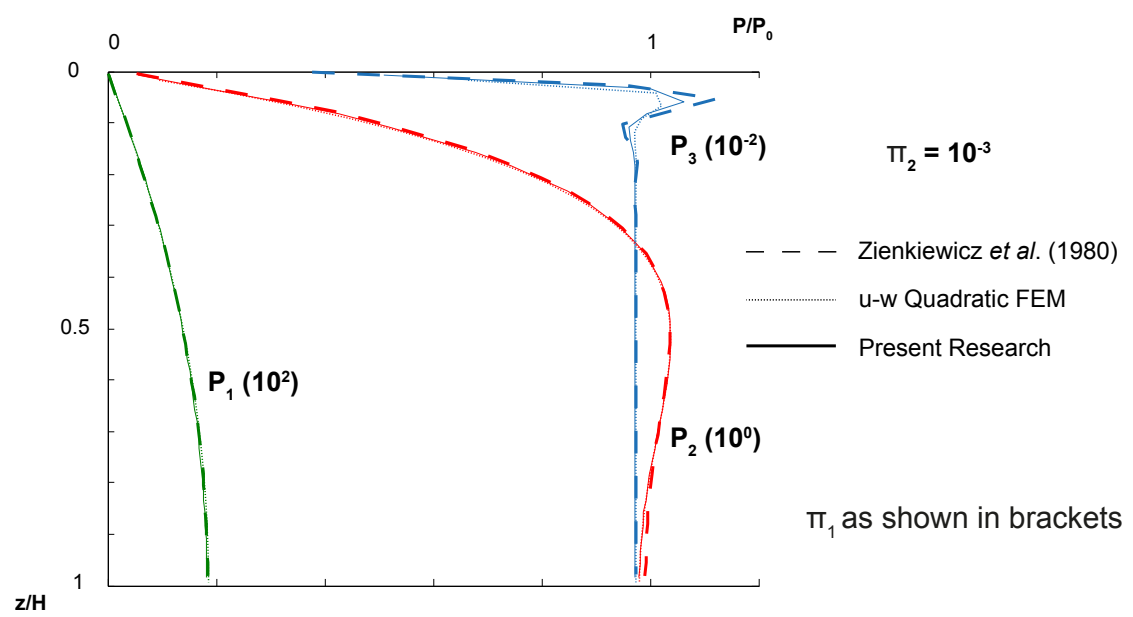

Figure 7: Isochrones of the pressure in the whole column for different $\pi_{1}$. The solution by Zienkiewicz et al [2] neglects the accelerations, while they are considered in the present research.

numerical results obtained with the current methodology fit reasonably well the analytical solutions in all cases. This demonstrates the robustness of the current approach for highly unstable consolidation problems, even in the range of high frequencies. No big differences are found with the solution of the finite element method. Even more, the meshfree solution fits better the analytical one for the highly unstable cases.

For both meshfree and finite element solutions, 320 integration points have been employed. However, despite of this fact, the performance is very different, the meshfree calculation being 6 or 7 times faster than the one obtained with finite elements. In Table 3 the performances of both, in terms of computational efforts, are presented. The computer employed had a processor: Intel Core i7 $2.3 \mathrm{GHz}$, with memory of $16 \mathrm{~GB} 1600 \mathrm{MHz}$. The software was MatLab R2014a. 


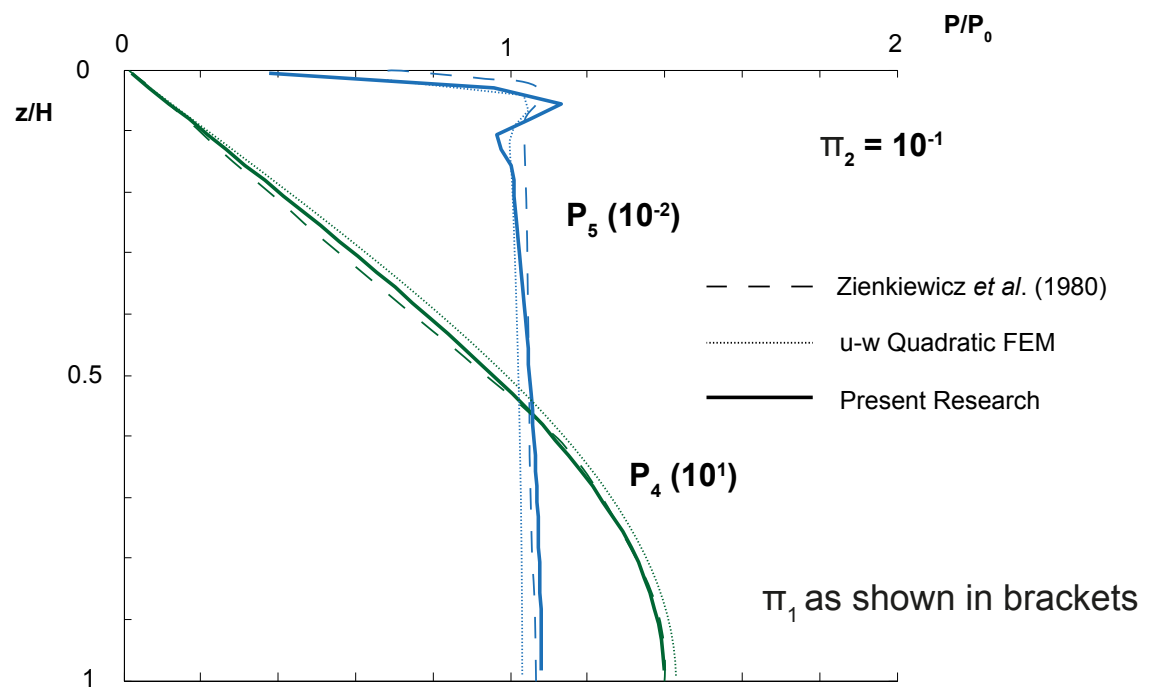

Figure 8: Comparison of the pressure isochrones obtained from the present research and the analytical one in the entire column for different values of $\Pi_{1}$, whereas $\Pi_{2}$ is kept constant.

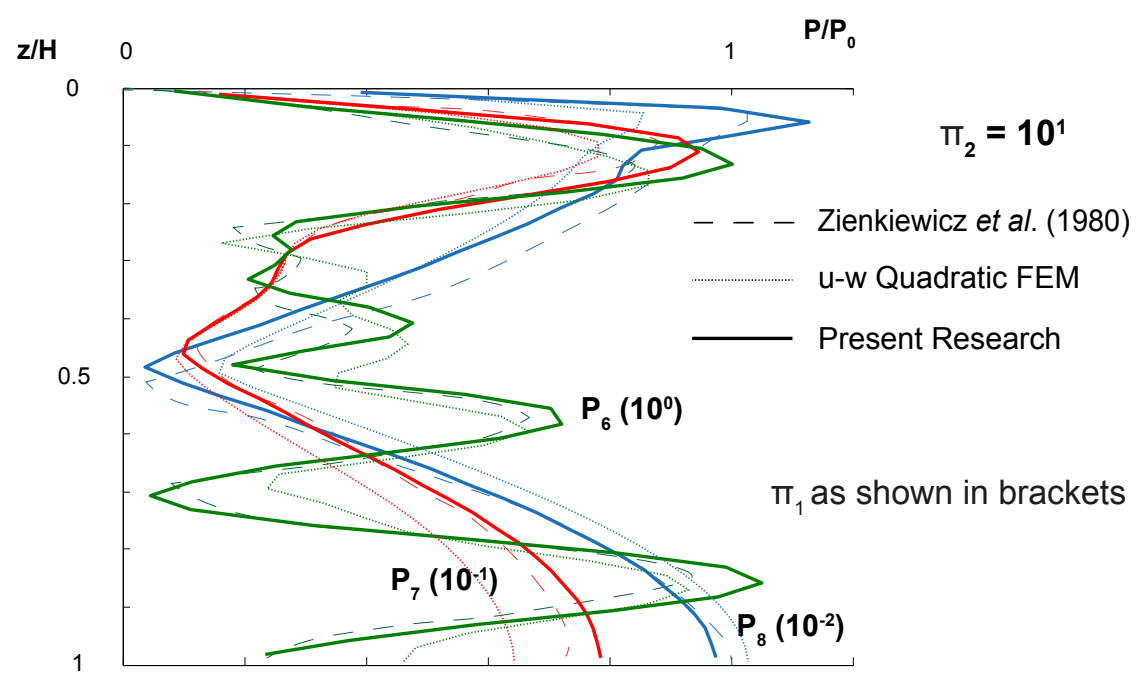

Figure 9: Comparison of the pressure isochrones obtained from the present research and the analytical one in the entire column for different $\Pi_{1}$, whereas $\Pi_{2}$ is kept constant. 


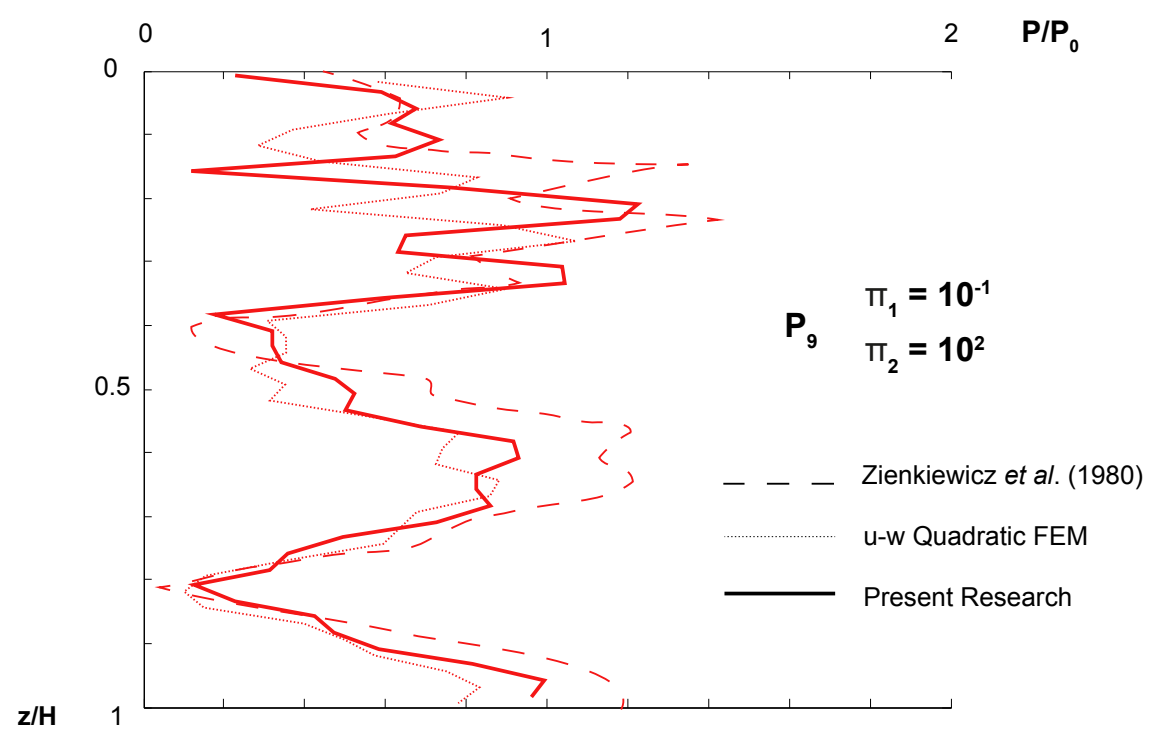

Figure 10: Comparison of the pressure isochrones obtained from the present research and the analytical one in the entire column for $\Pi_{1}=10^{-1}, \Pi_{2}=10^{2}$.

Table 3: Computational efforts, given in time of calculation (in seconds, $s$ ) with meshfree and finite elements simulations.

\begin{tabular}{c|ccccccccc}
\hline & $\mathrm{P} 1$ & $\mathrm{P} 2$ & $\mathrm{P} 3$ & $\mathrm{P} 4$ & $\mathrm{P} 5$ & $\mathrm{P} 6$ & $\mathrm{P} 7$ & $\mathrm{P} 8$ & $\mathrm{P} 9$ \\
\hline Meshfree & $26 \mathrm{~s}$ & $26 \mathrm{~s}$ & $26 \mathrm{~s}$ & $25 \mathrm{~s}$ & $26 \mathrm{~s}$ & $33 \mathrm{~s}$ & $26 \mathrm{~s}$ & $26 \mathrm{~s}$ & $65 \mathrm{~s}$ \\
Quad-FEM & $184 \mathrm{~s}$ & $188 \mathrm{~s}$ & $185 \mathrm{~s}$ & $185 \mathrm{~s}$ & $188 \mathrm{~s}$ & $188 \mathrm{~s}$ & $186 \mathrm{~s}$ & $190 \mathrm{~s}$ & $394 \mathrm{~s}$ \\
\hline Difference & $710 \%$ & $712 \%$ & $713 \%$ & $749 \%$ & $725 \%$ & $567 \%$ & $724 \%$ & $742 \%$ & $602 \%$
\end{tabular}


a)

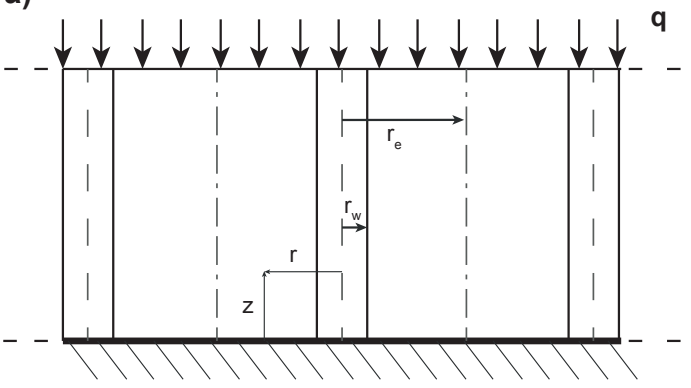

Impervious layer b)

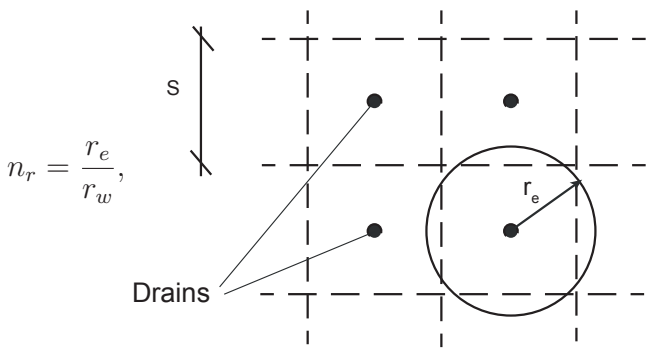

Figure 11: a) Scheme of section of set of drains and b) quadrangular net of drains $\left(\mathrm{r}_{e} / \mathrm{S}=0.564\right)$.

The physical equation governing the radial consolidation problem is different from the one given in the previous section. According to Terzaghi [30], it is as follows:

$$
c_{h}\left(\frac{\partial^{2} p_{w}}{\partial r^{2}}+\frac{1}{r} \frac{\partial p_{w}}{\partial r}\right)=\frac{\partial p_{w}}{\partial t}
$$

where $c_{h}$ is the horizontal consolidation coefficient. Since the radial consolidation equation involves a second term (tangential flow), it is impossible to solve it within a plane strain formulation, as the one employed for vertical one-dimensional consolidation. Therefore the axisymmetric framework shown in previous sections is utilised herein.

In Fig. 11, a sketch of drains with induced radial flow is presented, $r$ and $z$ representing the radial and vertical directions as depicted in the figure. $r_{w}$ is the drain radius and $r_{e}$ is the influence radius. Parameters of this soil are shown in Table 4.

Table 4: Material parameters employed for the radial consolidation problem.

\begin{tabular}{ccccc|cc|cc}
\hline $\begin{array}{c}\mathrm{E} \\
{[\mathrm{MPa}]}\end{array}$ & $\begin{array}{c}\nu \\
-\end{array}$ & $\begin{array}{c}\mathrm{n} \\
-\end{array}$ & $\begin{array}{c}\rho \\
{\left[\mathrm{kg} / \mathrm{m}^{3}\right]}\end{array}$ & $\begin{array}{c}\rho_{f} \\
{\left[\mathrm{~kg} / \mathrm{m}^{3}\right]}\end{array}$ & $\begin{array}{c}K_{f} \\
{[\mathrm{MPa}]}\end{array}$ & $\begin{array}{c}K_{s} \\
{[\mathrm{MPa}]}\end{array}$ & $\begin{array}{c}k \\
{[\mathrm{~m} / \mathrm{s}]}\end{array}$ & $\begin{array}{c}k_{\text {drain }} \\
{[\mathrm{m} / \mathrm{s}]}\end{array}$ \\
\hline 1.0 & 0.0 & 0.333 & 3003 & 1000 & $10^{4}$ & $10^{34}$ & $9.8 \mathrm{E}-3$ & $9.8 \mathrm{E}-1$ \\
\hline
\end{tabular}

The analytical solution for this problem was given by Barron in 1948 [33], [31]. Herein we study the case for a quadrangular net of drains as shown in 
Fig. 11b). In Fig. 12, several solutions of the radial consolidation degree, $U_{r}$, along the dimensionless time, $T_{r}$, are shown. Note that, except at the early stage, the solution obtained from the present methodology fits well the analytical ones.

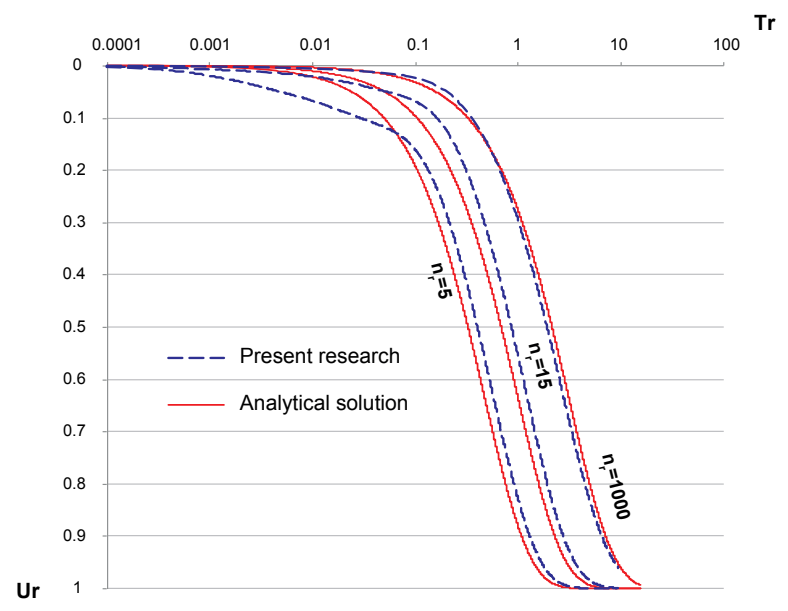

Figure 12: Analytical and numerical solutions of $U_{r}$ along the non-dimensional time $T_{r}$

A further comparison is carried out to validate the present methodology against the experimental results of Hsu and Liu [6] on radial consolidation. In the modelled test, the soil was vertically loaded with a pressure of $1.569 \mathrm{MPa}$, applied with a rate of $98.07 \mathrm{kPa} / \mathrm{h}$. The parameters and dimensions employed in the test are listed in Tab.5. The numerical solution is contrasted with both the analytical and the experimental solutions in Fig.13. Note that the analytical and numerical results fit very well for constant $c_{h}$ values. However, in order to obtain the solutions for varying $c_{h}$ (to represent the actual test conditions), a constitutive model to include plastic soil behaviour is indispensable, which is beyond the scope of the current work. In spite of that, these results show that the experimental tendency is correctly captured by the numerical simulations. Consequently, our numerical model is further validated with this example. 
Table 5: Parameters and dimensions employed in the radial consolidation test of Hsu and Liu $[6]$.

\begin{tabular}{cccc|ccc|cc}
\hline $\begin{array}{c}\mathrm{E} \\
{[\mathrm{MPa}]}\end{array}$ & $\begin{array}{c}\nu \\
-\end{array}$ & $\begin{array}{c}\mathrm{n} \\
{\left[\mathrm{kg} / \mathrm{m}^{3}\right]}\end{array}$ & $\begin{array}{c}c_{h} \\
{\left[\mathrm{~mm}^{2} / \mathrm{s}\right]}\end{array}$ & $\begin{array}{c}k_{h} \\
{[\mathrm{~mm} / \mathrm{s}]}\end{array}$ & $\begin{array}{c}k_{\text {drain }} \\
{[\mathrm{mm} / \mathrm{s}]}\end{array}$ & $\begin{array}{c}r_{e} \\
{[\mathrm{~mm}]}\end{array}$ & $\begin{array}{c}r_{w} \\
{[\mathrm{~mm}]}\end{array}$ \\
\hline 10.2 & 0.3 & 0.333 & 2000 & $1 \mathrm{E}-2$ & $1 \mathrm{E}-7$ & $1 \mathrm{E}-3$ & 31.75 & 8.75 \\
\hline
\end{tabular}

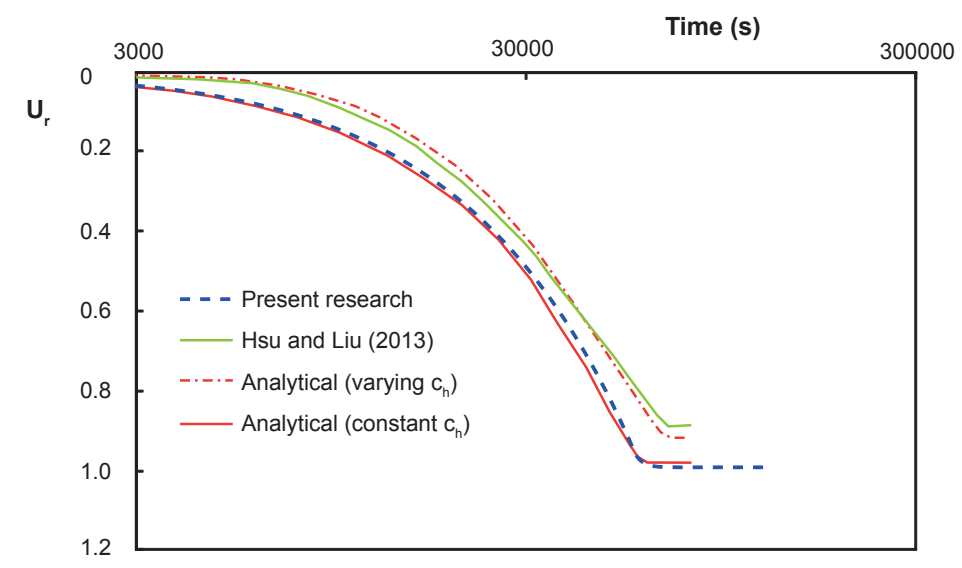

Figure 13: Analytical, experimental and numerical solutions for the radial consolidation test of Hsu and Liu [6].

\subsection{Radial consolidation: dynamic axisymmetric problem}

In order to deal with the dynamic radial consolidation problem, a dynamic loading at the surface has been applied to the same geometry as in Fig. 11. The dynamic effect on the development of excess pore water pressure in the domain is studied. Similarly to the 1D dynamic consolidation problems, various scenarios representing different zones of behaviour in Fig. 6b) are explored herein. The loading frequency and the hydraulic conductivity in Table 6, are chosen to give the predefined hypothetical dimensionless parameters, taking into account that the term, $H_{T}$, in Eq. (33) refers now to the horizontal distance to the drain. Four simulations, denoted as A, C, D and E in Fig. 6b, have been carried out. Vertical displacements of water in the entire domain are prevented to reproduce a purely radial process. 
Table 6: Angular velocity and permeability for each of the four radial dynamic consolidation problems.

\begin{tabular}{l|ll|lc}
\hline & $\Pi_{1}$ & $\Pi_{2}$ & $k[\mathrm{~m} / \mathrm{s}]$ & $\omega[\mathrm{rad} / \mathrm{s}]$ \\
\hline $\mathrm{A}$ & $10^{-1}$ & $10^{-2}$ & $5.82 \times 10^{-5}$ & 56.07 \\
$\mathrm{C}$ & $10^{-2}$ & $10^{0}$ & $5.82 \times 10^{-5}$ & 560.7 \\
$\mathrm{D}$ & $10^{-1}$ & $10^{2}$ & $5.82 \times 10^{-3}$ & 5607 \\
$\mathrm{E}$ & $10^{2}$ & $10^{2}$ & $5.82 \times 10^{0}$ & 5607 \\
\hline
\end{tabular}

The first row of Fig. 14 represents the maximum envelope of the horizontal isochrone of excess pore pressure at three different elevations along the domain $(0.5 \mathrm{~m}, 5.0 \mathrm{~m}$ and $9.5 \mathrm{~m})$. As expected, high frequency problems (with higher $\Pi_{2}$ values), D and $\mathrm{E}$, have a more unstable behaviour than the low frequency one, A. In addition, since $\Pi_{1}$ is higher for E, thus presents higher peaks. The second row shows the same results along three different radial distances from the drain $(0.5 \mathrm{~m}, 3 \mathrm{~m}$ and $5.0 \mathrm{~m})$. In this case, the difference between $\mathrm{D}$ and $\mathrm{E}$ is inappreciable, although both are significantly different from A. However, for case C (located in Zone II in Fig. 6), a significant overpressure at the bottom of the column is observed, which was seen in the first row as well. This can be attributed to the fact that when the instability of the water pressure arrives at the bottom of the soil but is not reflected, since the soil is not permeable enough so that the water movement may be significant. Similar behaviour is perceived in P4, P7 and P8 in Figs. 8-9. In the third row of Fig. 14, the evolution of pore water pressure at three different elevations of the column at the radius of $0.5 \mathrm{~m}$ are illustrated for case $\mathrm{A}$ and $\mathrm{C}$, whereas maximum and minimum envelope evolution with time are shown for cases D and E. It needs to be emphasised that the steady state is achieved for case A at the first cycle of loading, whereas for case $\mathrm{C}$ the convergence is relatively fast and for cases $\mathrm{D}$ and $\mathrm{E}$, the solutions become stable after $0.08 \mathrm{~s}$. The maximum and minimum values of excess pore water pressure in the entire domain at steady state are shown for A and D respectively in Fig. 15 and Fig. 16. Note that in contrast to Fig. 15, low frequency cases present a slow pressure redistribution, which is similar to a series of static states. By contrast, the high-frequency results, typically show a pattern that depicts the distributions of the waves. Indeed, from the alternate feature shown in Fig. 16, the position of the drain is not detectable, consequently, it might exert litter influence over the pressure distribution. 

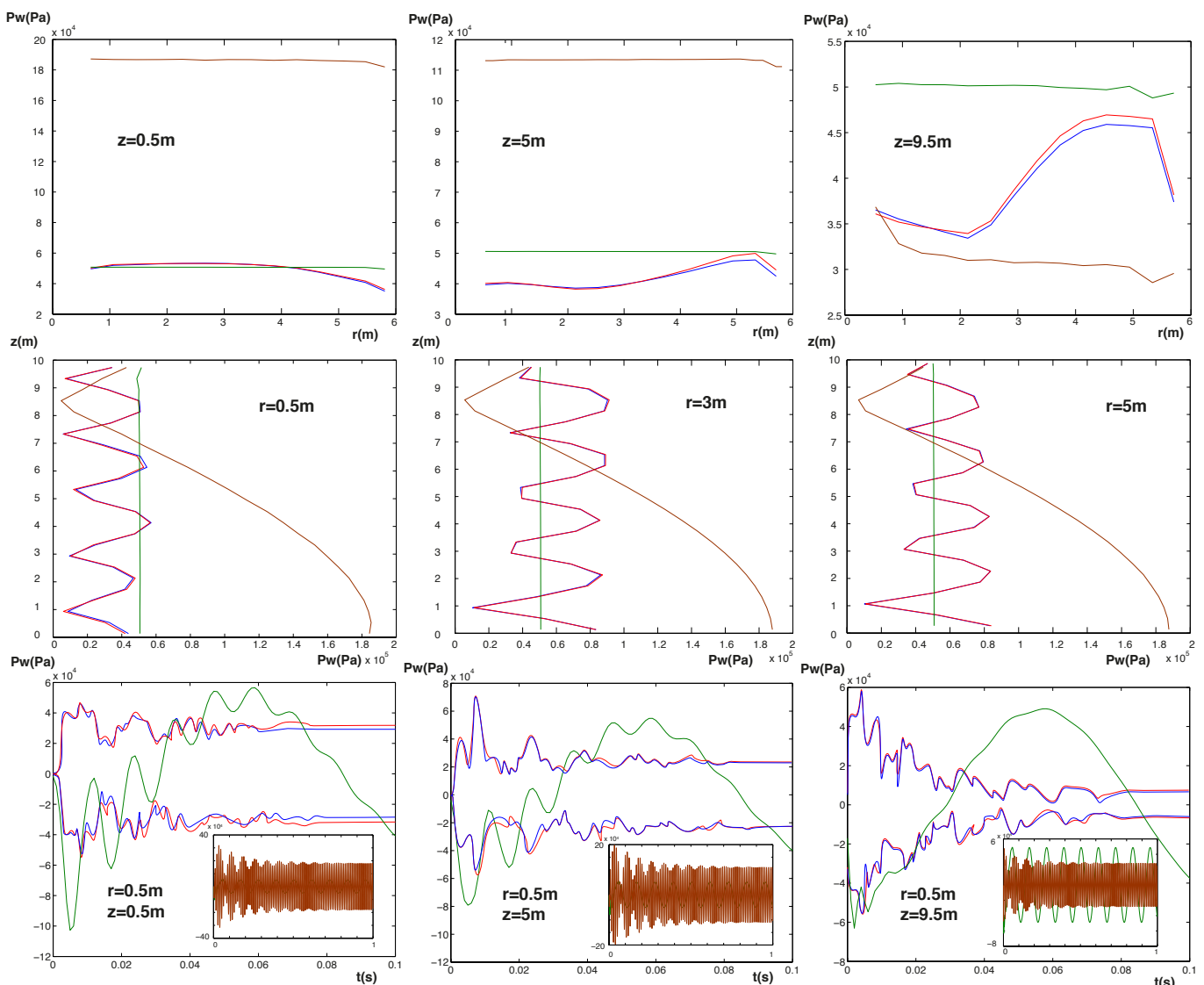

A) $\pi_{2}=10^{-2}, \pi_{1}=10^{-1}$

C) $\pi_{2}=10^{\circ}, \pi_{1}=10^{-2}$

D) $\pi_{2}=10^{2}, \Pi_{1}=10^{-1}$

E) $\pi_{2}=10^{2}, \pi_{1}=10^{2}$

Figure 14: Excess pore water distribution at three different heights $0.5 \mathrm{~m}, 5.0$ and $9.5 \mathrm{~m}$ (top row); at distances of $0.5 \mathrm{~m}, 3 \mathrm{~m}$ and $5 \mathrm{~m}$ from the sink (middle row); and evolution of excess pore water pressure with time at different locations $(0.5,0.5),(0.5,5.0)$ and $(0.5$, 9.5), for cases $\mathrm{A}$ and $\mathrm{C}$ and corresponding envelopes for cases $\mathrm{D}$ and $\mathrm{E}$. 

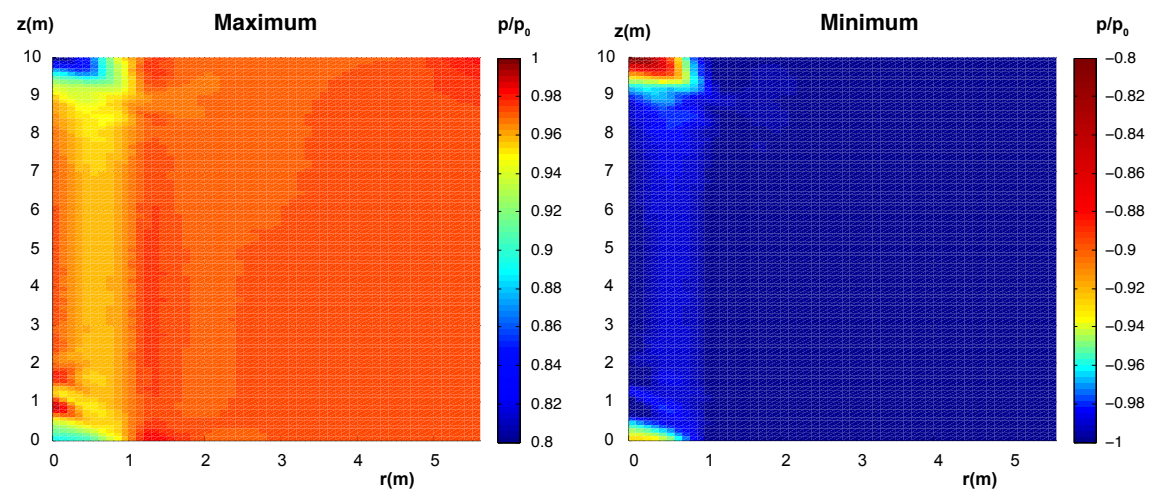

Figure 15: Maximum and minimum normalised excess pore water pressures at steady state for dynamic, radial consolidation problem, case A defined in Table 6 .

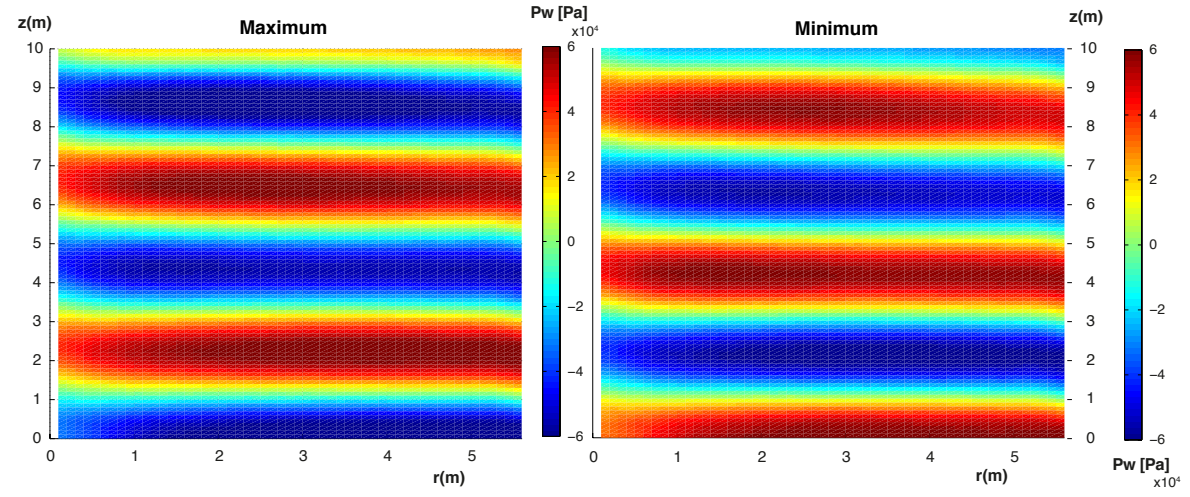

Figure 16: Maximum and minimum excess pore water pressures at steady state for dynamic, radial consolidation problem, case D defined in Table 6. 


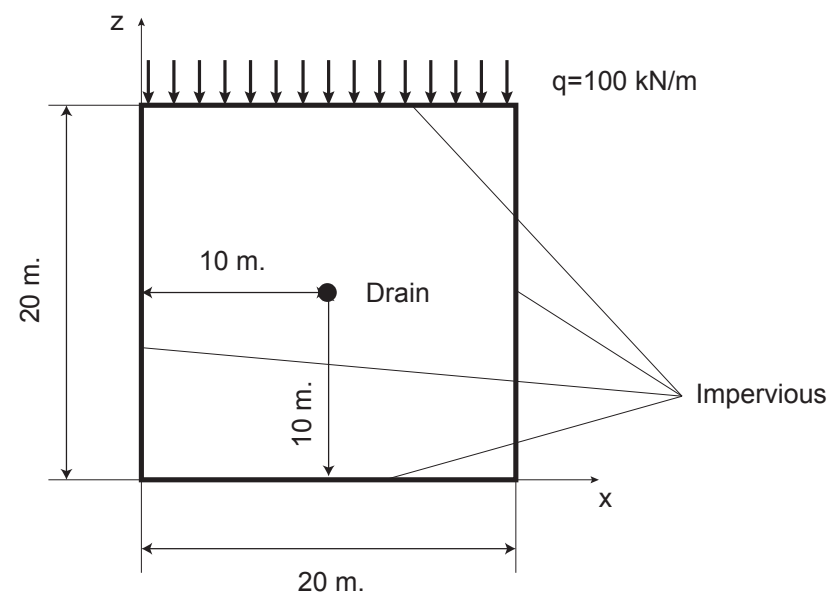

Figure 17: Geometry for the soil consolidation problem with a sink.

\subsection{Static consolidation in a soil with a singular drainage point: the static sink problem}

In this Section, we apply the previously developed approach to model the soil consolidation problem when a singular drainage point is introduced in the domain. The existence of the sink is expected to accelerate the consolidation of the porous media, since there is an additional output of flow around the sink point. To reproduce this singular drainage point, excess pore water pressure is impeded to develop at several nodes around the domain center. The simulated geometry is a square section with 20-meter edge length, see Fig. 17. The employed material properties are given in Table 7.

The evolution of the consolidation degree, $U$, at the bottom, lowest corner and the $U$-distribution over the entire domain after two seconds are respectively plotted in Fig. 18 and Fig. 19. In spite of slight differences in the final part of the evolution in Fig. 18, it can be concluded that the results from the current approach and the commercial software are fairly similar, thus validating the present formulation for this kind of problems.

Table 7: Material parameters employed for the soil consolidation problem with a sink.

\begin{tabular}{ccccc|cc|cc}
\hline $\begin{array}{c}\mathrm{E} \\
{[\mathrm{MPa}]}\end{array}$ & $\begin{array}{c}\nu \\
-\end{array}$ & $\begin{array}{c}\mathrm{n} \\
{\left[\mathrm{kg} / \mathrm{m}^{3}\right]}\end{array}$ & $\begin{array}{c}\rho_{f} \\
{\left[\mathrm{~kg} / \mathrm{m}^{3}\right]}\end{array}$ & $\begin{array}{c}K_{f} \\
{[\mathrm{MPa}]}\end{array}$ & $\begin{array}{c}K_{s} \\
{[\mathrm{MPa}]}\end{array}$ & $\begin{array}{c}k \\
{[\mathrm{~m} / \mathrm{s}]}\end{array}$ & $\begin{array}{c}k_{\text {sink }} \\
{[\mathrm{m} / \mathrm{s}]}\end{array}$ \\
\hline 100 & 0.0 & 0.333 & 3003 & 1000 & $10^{3}$ & $10^{34}$ & $10^{-3}$ & 10 \\
\hline
\end{tabular}




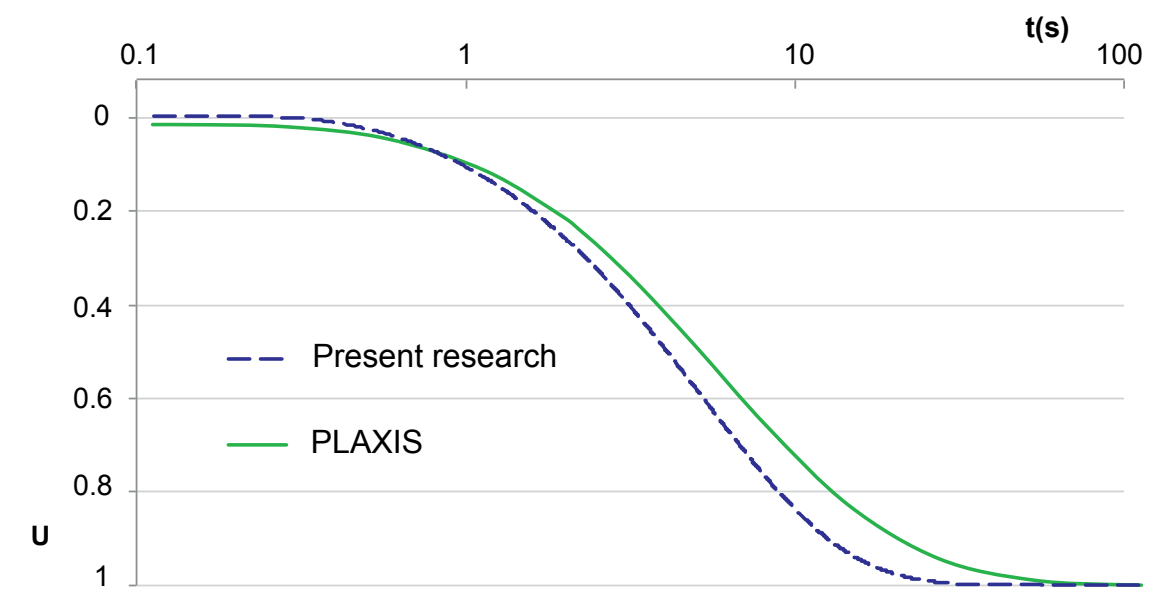

Figure 18: Comparison of the evolution of consolidation degree at the left bottom corner of the domain for the sink problem. Present model vs. PLAXIS.
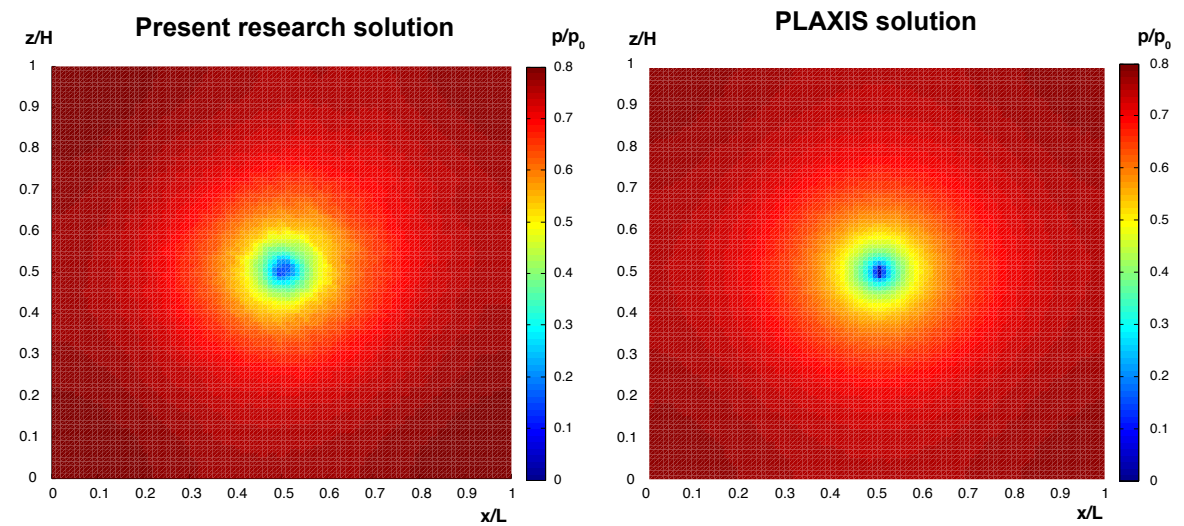

Figure 19: Field of consolidation degrees in the domain after 2 seconds. Present model vs. PLAXIS. 
Table 8: Angular velocity and permeability in each of the three sink dynamic consolidation problems.

4.6. Dynamic consolidation in a soil with a singular point: the dynamic sink problem

\begin{tabular}{l|ll|ll}
\hline & $\Pi_{1}$ & $\Pi_{2}$ & $k[\mathrm{~m} / \mathrm{s}]$ & $\omega[\mathrm{rad} / \mathrm{s}]$ \\
\hline $\mathrm{A}$ & $10^{-1}$ & $10^{-2}$ & $62.25 \times 10^{-5}$ & 5 \\
$\mathrm{C}$ & $10^{-2}$ & $10^{0}$ & $62.25 \times 10^{-5}$ & 50 \\
$\mathrm{D}$ & $10^{-1}$ & $10^{2}$ & $62.25 \times 10^{-3}$ & 500 \\
$\mathrm{E}$ & $10^{2}$ & $10^{2}$ & $62.25 \times 10^{0}$ & 500 \\
\hline
\end{tabular}

In this Section, we study the dynamic counterpart of the consolidation problem presented in Section 4.5. The same geometry in Fig. 17 is opted for and the material properties in Table 7 are employed. Taking into account Eq. (33), the permeability coefficients and angular velocities in Table 8 are selected to give the corresponding dimensionless parameters $\Pi_{1}$ and $\Pi_{2}$ for the points A, C, D and E defined in Fig.6b).

In Fig. 20, the evolution of excess pore water pressure at the top right corner for all three cases are plotted. For the sake of clarity, maximum and minimum envelopes are illustrated. As mentioned before, the higher the $\Pi_{2}$, the more unstable the evolution of the excess pore pressure. Consequently, the slowest (fastest) convergence and highest (lowest) pressure amplitudes are presented for case E (A) before the steady state is achieved. The same trend is observed in Fig. 21 where different peak values occur along the depth for high frequency problems $\mathrm{D}$ and $\mathrm{E}$, whereas uniform amplitude is obtained for the low frequency case A. The intermediate case $\mathrm{C}$, overpressure at the bottom of the domain is observed, this is similar to the case $\mathrm{C}$ of the radial consolidation problem shown in Fig. 14. Note that unreasonable pore pressure values near the sink are cut off from the figure. Furthermore, in Fig. 22, the maximum and minimum excess pore water pressures over the entire domain are depicted for both the low frequency case A and high frequency cases D, E. Once again, these results demonstrate the suitability of the present formulation for dynamic consolidation problems in saturated soils. 

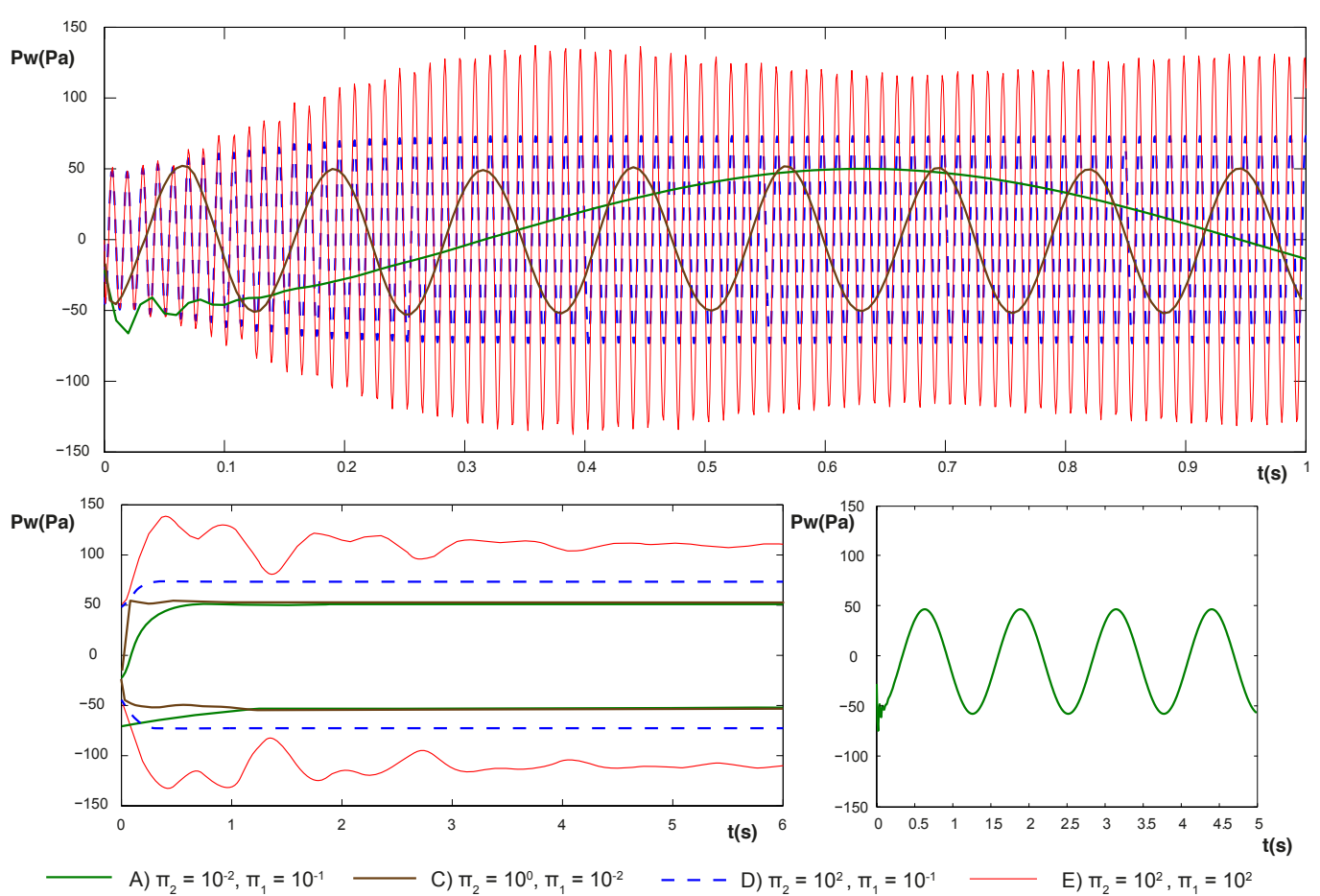

Figure 20: Evolution of excess pore water pressure during external cyclic loading at the top, right corner (for the sake of clarity, the left, lower figure represents maximum and minimum envelope of the solutions).
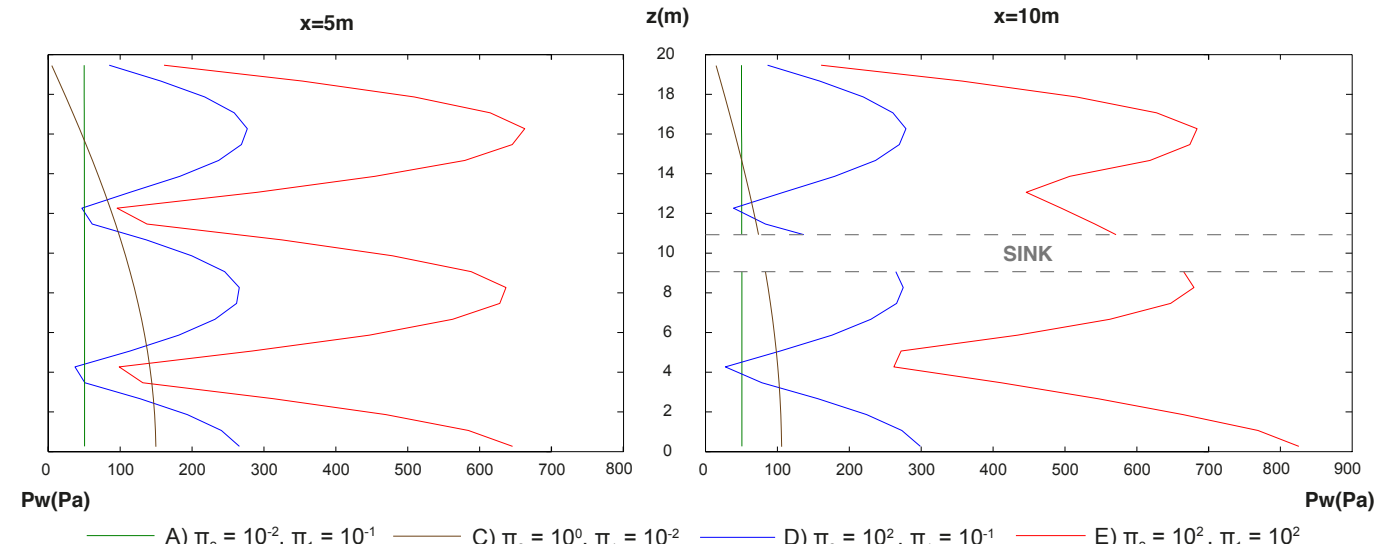

Figure 21: Maximum isochrones of the excess pore water pressure along two columns of the domain ( $5 \mathrm{~m}$ and $10 \mathrm{~m}$ from the left border). 


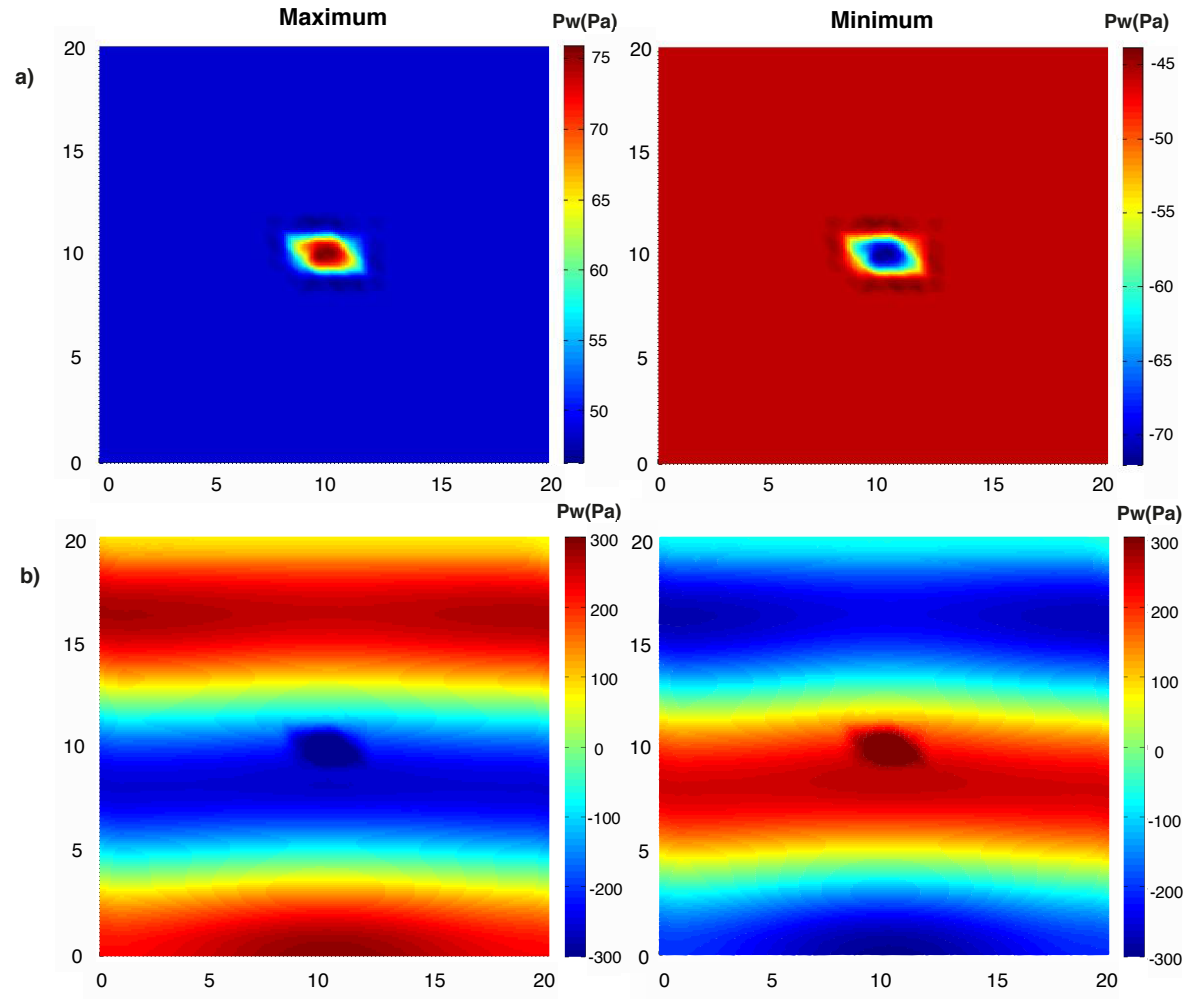

Figure 22: Maximum and minimum excess pore water pressures in dynamic consolidation with a sink for a) the low frequency problem, and b) the high frequency problem. 

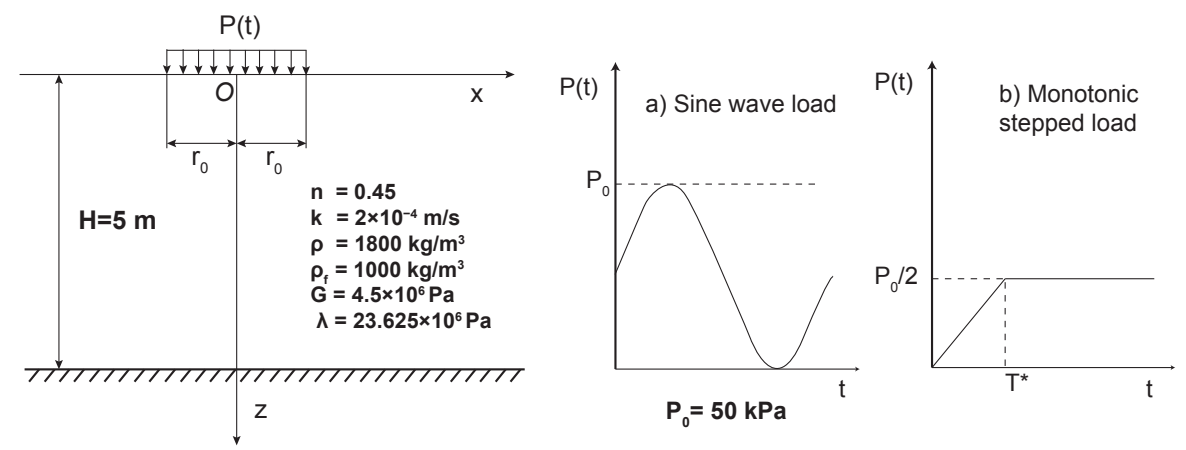

Figure 23: The Lamb's problem: geometry, material parameters and loading (static and dynamic).

\subsection{Axisymmetric Lamb's problem}

The propagation of vibrations over the surface of a semi-infinite isotropic elastic solid was first studied by Lamb [34] in 1904. Since a saturated soil needs to be treated as a two-phase medium which consists of soil skeleton and pore water, the coupled problem has been dealt with as a traditional problem of consolidation in porous media, see [35, 36, 37], or through an axisymmetric scheme to obtain the solution in a more realistic situation, see [38]. Herein we tackle the Lambs problem using the axisymmetric meshfree formulation validated in Section 4.5. In order to compare our results with those of Cai et al. [38], the same geometry and material parameters as shown in Fig. 23 are employed. Additionally plotted in Fig. 23 are the harmonic and stepped loading for the two series of transient calculations carried out.

It needs to be pointed out that, since a $u-w$ formulation is assumed in the current work, the total water displacement in the vertical direction is extracted as

$$
U_{z}=u+\frac{w}{n} .
$$

The obtained results for three different levels of permeability are compared with those of Cai et al. [38] for the case of stepped loading, with ramped time $T^{*}$ of one second, in Fig. 24. Close agreement is achieved for all three cases.

For the case of harmonic loading, the dimensionless parameter

$$
a_{0}=\frac{\omega r_{0}}{V_{s}}, \quad \text { where } V_{s}=\sqrt{\frac{G}{\rho}} .
$$

which was defined by Cai et al. [38], is adopted to characterise the combined effect of loading frequency and load area. Four simulations, represented 


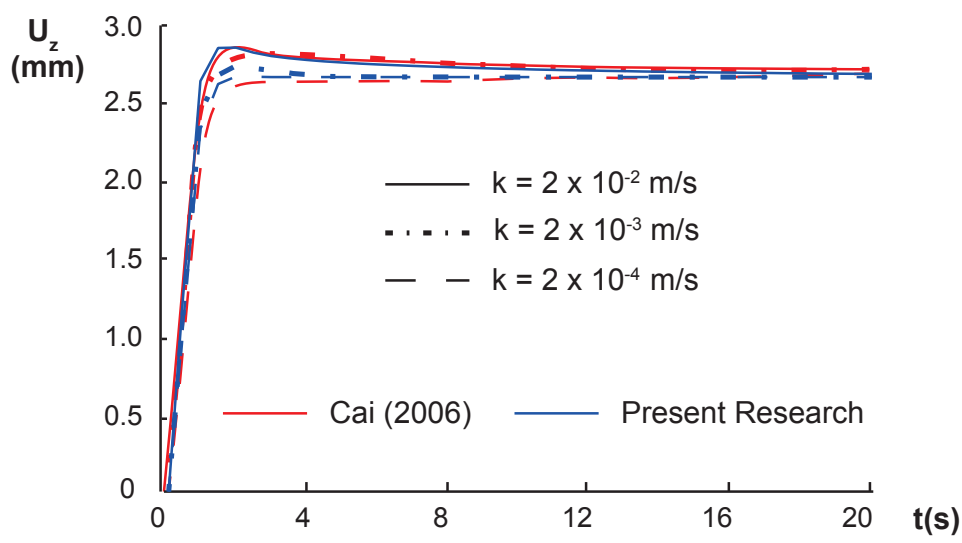

Figure 24: Lamb's problem water vertical displacement of saturated soil subjected to gradually applied stepped load $\left(T^{*}=1\right)$.

Table 9: Angular velocity and permeability in each of the Lamb's problems.

\begin{tabular}{l|clr|rcr}
\hline & $\Pi_{1}$ & $\Pi_{2}$ & $a_{0}$ & $r_{0}[\mathrm{~m}]$ & $k[\mathrm{~m} / \mathrm{s}]$ & $\omega[\mathrm{rad} / \mathrm{s}]$ \\
\hline $\mathrm{A}$ & $10^{-1}$ & $10^{-2}$ & 0.4 & 1.0 & $2 \times 10^{-4}$ & 20 \\
$\mathrm{~B}$ & $10^{-1}$ & $10^{-2}$ & 4.0 & 10.0 & $2 \times 10^{-4}$ & 20 \\
$\mathrm{C}$ & $10^{-2}$ & $10^{0}$ & 4.0 & 1.0 & $2 \times 10^{-4}$ & 200 \\
$\mathrm{D}$ & $10^{-1}$ & $10^{2}$ & 40.0 & 1.0 & $2 \times 10^{-2}$ & 2000 \\
\hline
\end{tabular}


by points, A, B, C and D, as marked in Fig. 6b) and the corresponding parameters listed in Table 9 are carried out. In Fig. 25, the envelope of maximum displacements is represented for a total computation time of 20 seconds. It needs to be remarked that, even though points $\mathrm{A}$ and $\mathrm{B}$ coincide in Fig. 6b), the loading area for point $\mathrm{A}$ is only one percent of that for point $\mathrm{B}$, consequently, the vertical displacement is more extended for case B.
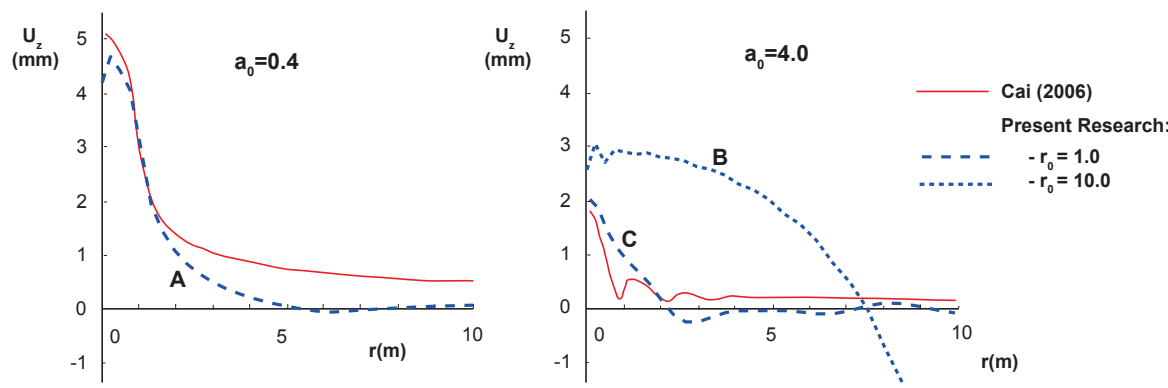

Figure 25: Envelopes of vertical water displacements along the radial direction during $20 \mathrm{~s}$ for cases $\mathrm{A}, \mathrm{B}$ and $\mathrm{C}$.

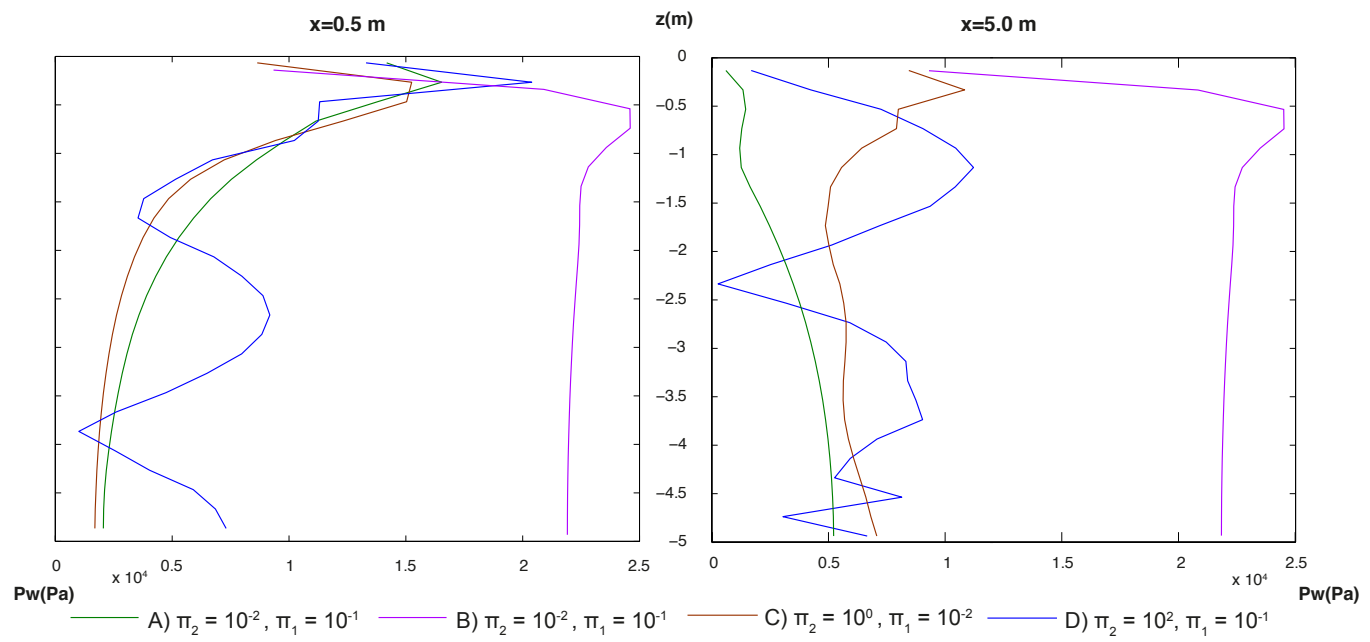

Figure 26: Maximum envelope of isochrones of the pore pressure along two columns in the domain. 

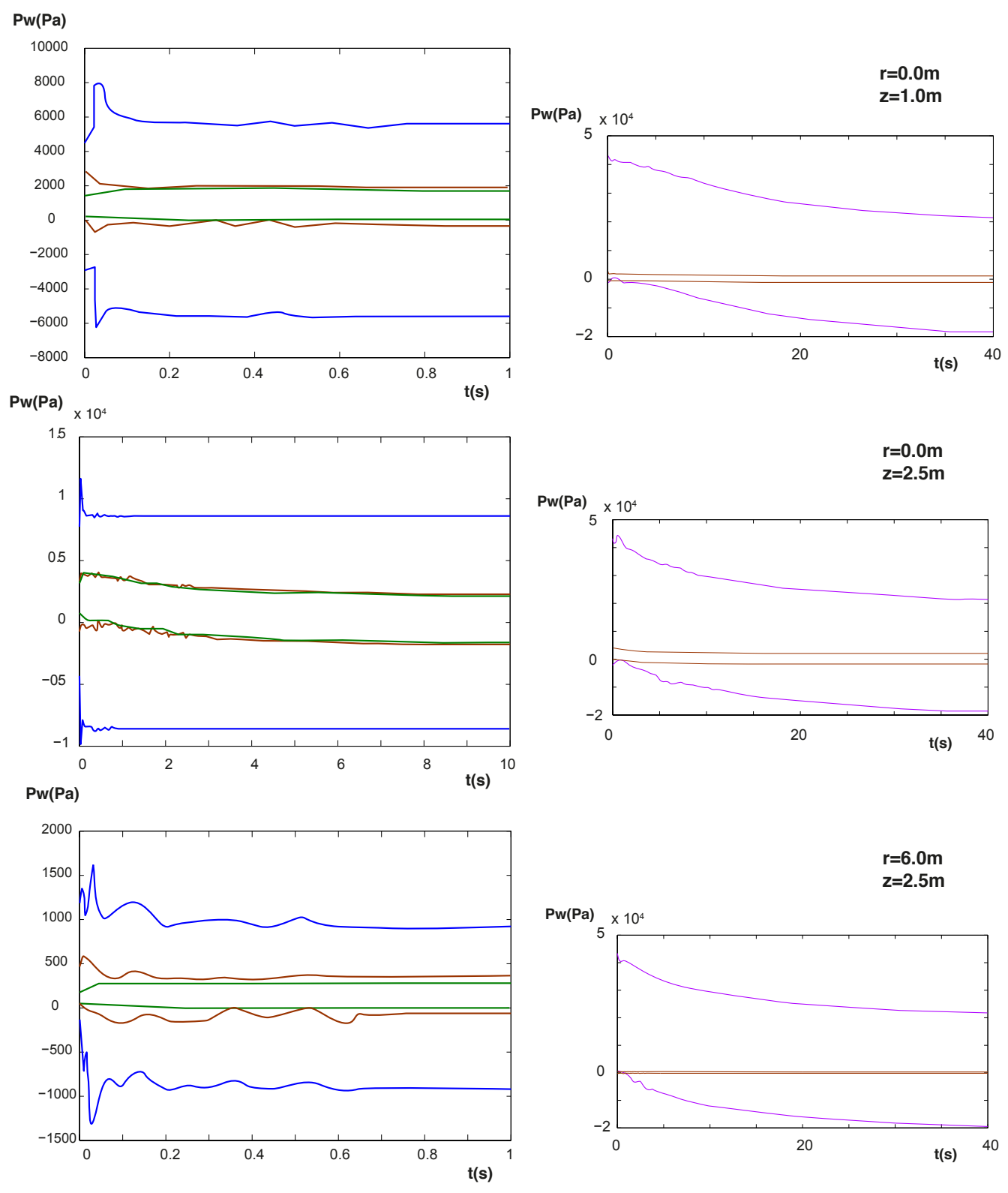

A) $\pi_{2}=10^{-2}, \pi_{1}=10^{-1}-$ B) $\pi_{2}=10^{-2}, \pi_{1}=10^{-1}$

C) $\pi_{2}=10^{\circ}, \pi_{1}=10^{-2}$

D) $\pi_{2}=10^{2}, \pi_{1}=10^{-1}$

Figure 27: Evolution of maximum and minimum envelopes of excess pore water pressure during external cyclic loading at three different locations: $(0.0,1.0),(0.0,2.5)$ and $(6.0,2.5)$ for A, B, C and D.

In Fig. 26, the maximum envelopes of isochrones of the pore pressure 

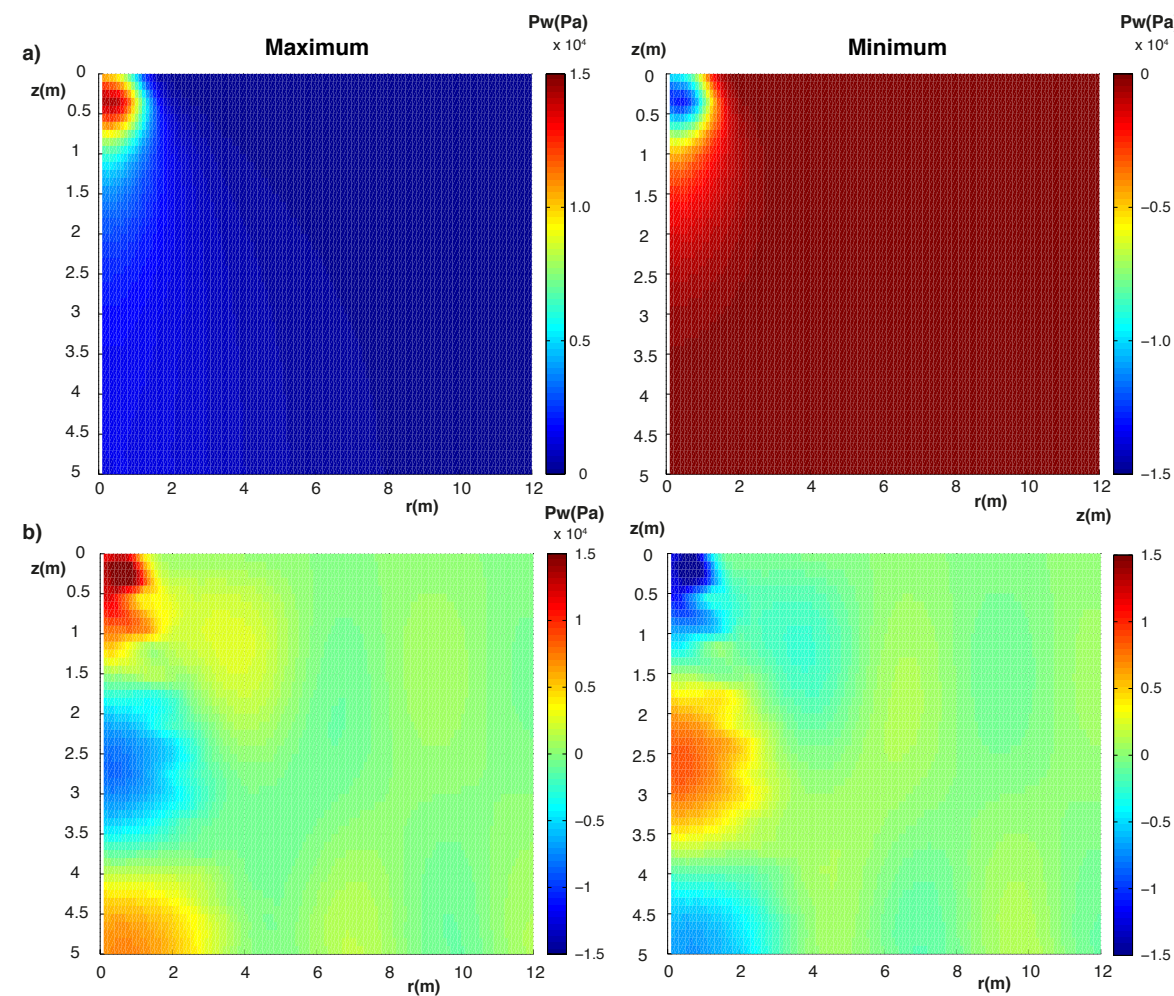

Figure 28: Maximum and minimum pore pressure distribution within a) slow (case A) and b) fast (case D) Lamb's problem. 
are compared for cases A, B, C and D. Note that A, C and D share the same loading radius of $1 \mathrm{~m}$, but represent the low $(20 \mathrm{rad} / \mathrm{s})$, medium $(200 \mathrm{rad} / \mathrm{s})$ and high (2000 rad/s) frequencies, and fall in Zone I, Zone II and Zone III respectively. By contrast, case B refers to a wider loading area (a radius of 10 $\mathrm{m})$. The total histories of the pressure envelopes at three different locations in the domain for all four cases are represented in Fig. 27. Note that a different scale is used for case B, since the pressure amplitudes are much higher due to a wider loading area, see Figs. 26-27. Similar patterns seen in the previous can be noticed in the A, C and D cases. In general, high-frequency problems present higher pressure peaks and more instabilities than low-frequency ones. Similar peak values are obtained for A and C, but the convergence is faster for case $\mathrm{A}$ as the frequency is lower. Furthermore, the maximum and minimum pressures of the entire domain under low (case A) and high (case D) frequency situations are depicted in Fig. 28. It is noteworthy the unstable behaviour of the fast frequency results are well captured using the current formulation. Note the different zones where the maximum and minimum values in Fig. 26 and Fig. (28)b, which are attributed to the reflection of the waves at the bottom boundary in the fast problems. This fact does not appear in slow problems because there is enough time to dissipate the excess pore pressure.

\section{Conclusions}

We have extended the previously developed B-bar based algorithm to mesh-free numerical schemes in axisymmetric framework for porous media. The methodology is applied to both static and dynamic consolidation problems in saturated soils. Static and dynamic consolidation of a soil column, radial consolidation, consolidation with singular points (sinks), as well as the Lamb's problem, have been simulated and compared with analytical solutions (whenever they exist) or available solutions obtained with finite element based codes. The feasibility of the current formulation in solving consolidation problems in saturated soils, particularly for dynamic ones in high frequency domain, has been clearly demonstrated. The better efficiency, in terms of computational efforts, compared with FE simulations has also been demonstrated for dynamic cases. Moreover, applications of the LME meshfree approximation in axisymmetric soil consolidation problems using $u-w$ formulation have not been previously reported in the literature.

In addition, the numerical results presented in this paper for high-frequency dynamic problems are completely new and the feasibility of the developed methodology is rather promising even for such unstable cases. It is verified that the complete $u-w$ formulation is particularly suitable for the modelling of dynamic high-frequency problems, which had been previously 
demonstrated in finite element approaches, but never before in meshfree models for soils.

The novelty of the current work also lies in the presentation of the soil behaviour for different drainage configurations and under different types of loading. Results are shown both along time and at representative locations of the domain, paying special attention to the peak values. The employment of behaviour classification as for the zones given by Zienkiewicz et al. [2], illustrated in Fig. 6, is adopted for all the problems studied in this paper. Although slight differences are noticed, the pattern behaviour of the soil is closely related with the mentioned figure in all cases. Cases in zone I present a quasi-static behaviour, as the pore pressure is redistributed with a fast convergence along the domain. In zone II, the high-moderate frequency leads the acceleration of the soil being important, causing several instabilities, although, as Zienkiewicz stated, the acceleration of the water phase can be neglected due to the low permeability, which normally provokes an overpressure at the bottom. The bigger the values of $\Pi_{1}$ and $\Pi_{2}$, the more unstable and higher peaks of the pore pressure. As the permeability is high, distributions of the waves along the domain are observed and the accelerations of the fluid phase become essential if an accurate and stable solution is to be found.

The radial consolidation is an axisymmetric problem, but as in the consolidation of a soil column, in terms of water displacement, it is a $1 \mathrm{D}$ problem, in which only horizontal water movement is allowed. The classification in zones as proposed by Zienkiewicz et al. [2] is demonstrated to be valid also in this problem, in which cases located in the same zones behave in a quite similar manner and same range of instability.

In addition, the sink problem is purely 2D, with vertical and horizontal displacements in both solid and fluid phases. In this case, although Zienkiewicz's classification is still approximate, some differences appear in problems located in zone III: the higher the $\Pi_{1}$, the higher the amplitude in the response in terms of pore water pressures, and the longer it takes until a steady solution is found. The same trend was also found in the Lamb's problem, also 2D in nature. This behaviour was not obtained in the case of radial consolidation.

Finally, as the unstable behaviour of high-frequency problems is very well captured by the employed formulation, which stresses the robustness of this methodology. Further study on this topic is still needed to seek out the limit response of the soil under high frequency loadings. Implementation of plastic constitutive laws under the current meshfree framework deserves further study.

Comparison between the present formulation and experimental research, 
taking into account non elastic soil, would be required in the future to extend the validity of this new methodology to real consolidation problems.

\section{Acknowledgements}

The financial support to develop this research from the Ministerio de Ciencia e Innovación, under Grant Numbers, BIA2012-31678 and MAT201235416, and the Consejería de Educación, Cultura y Deportes de la Junta de Comunidades de Castilla-La Mancha, Fondo Europeo de Desarrollo Regional, under Grant No. PEII-2014-016-P, Spain, is greatly appreciated. The first author also acknowledges the fellowship BES2013-0639 received.

[1] M. A. Biot. General theory of three-dimensional consolidation. Journal of Applied Physics, 12(2):155-164, February 1941.

[2] O.C. Zienkiewicz, C.T. Chang, and P. Bettes. Drained, undrained, consolidating and dynamic behaviour assumptions in soils. Géotechnique, 30(4):385-395, 1980 .

[3] M. A. Biot. General solutions of the equations of elasticity and consolidation for a porous material. Journal of Applied Mechanics, pages 91-96, March 1956.

[4] S. López-Querol and R. Blázquez. Liquefaction and cyclic mobility model in saturated granular media. International Journal for Numerical and Analytical Methods in Geomechanics, 30:413-439, 2006.

[5] A. Cividini and G. Gioda. On the dynamic analysis of two-phase soils. In S. Pietruszczak and G. N. Pande, editors, Proceedings of the Third International Symposium on Computational Geomechanics (ComGeo III), pages 452-461, 2013.

[6] T.W. Hsu and H.J. Liu. Consolidation for radial drainage under timedependent loading. Journal of Geotechnical and Geoenvironmental Engineering, 139(12):2096-2103, 2013.

[7] Y.Y. Hu, W.H. Zhou, and Y.Q. Cai. Large-strains elastic viscoplastic consolidation analysis of very soft clay layers with vertical drains under preloading. Canadian Geotechnical Journal, 51:144-157, 2014.

[8] S. Basack, B. Indraratna, and C. Rujikiatkamjom. Modeling the performance of stone column-reinforced soft ground under static and cyclic loads. Journal of Geotechnical and Geoenvironmental Engineering, 142(2:04015067):115, 2016. 
[9] P. Navas, S. López-Querol, R.C. Yu, and B. Li. B-bar based algorithm applied to meshfree numerical schemes to solve unconfined seepage problems through porous media. International Journal for Numerical and Analytical Methods in Geomechanics, DOI:10.1002/nag.2472, October 2015.

[10] A. Ortiz, M.A. Puso, and N. Sukumar. Construction of polygonal interpolants: A maximum entropy approach. International Journal for Numerical Methods in Engineering, 61(12):2159-2181, 2004.

[11] T.J.R. Hughes. Generalization of selective integration procedures to anisotropic and nonlinear media. International Journal for Numerical Methods in Engineering, 15:1413-1418, 1980.

[12] J.C. Simo and M.S. Rifai. A class of mixed assumed strain methods and the method of incompatible modes. International Journal for Numerical Methods in Engineering, 29:1595-1638, 1990.

[13] E.P. Kasper and R.L. Taylor. A mixed-enhanced strain method: Part I: Geometrically linear problems. Computers and Structures, 75(3):237-250, 2000 .

[14] E.A. De Souza Neto, F.M. Pires, and D.R.J. Owen. F-bar-based linear triangles and tetrahedra for finte strain analysis of nearly incompressible solids. Part I: formulation and benchmarking. International Journal for Numerical Methods in Engineering, 62:353-383, 1980.

[15] J. Bonet and A.J. Burton. A simple average nodal pressure tetrahedral element for incompressible and nearly incompressible dynamic explicit applications. Communications in Numerical Methods in Engineering, 14(5):437-449, May 1998.

[16] P. Hauret, E. Kuhl, and M. Ortiz. Diamond elements: A finite element/discrete-mechanics approximation scheme with guaranteed optimal convergence in incompressible elasticity. International Journal for Numerical Methods in Engineering, 73:253-294, 2007.

[17] T. Elguedj, Y. Bazilevs, V. M. Calo, and T.J.R. Hughes. $\bar{B}$ and $\bar{F}$ projection methods for nearly incompressible linear and non-linear elasticity and plasticity using higher-order NURBS elements. Computer Methods in Applied Mechanics and Engineering, 197(33-40):2732-2762, 2008.

[18] E. Artioli, G. Castellazzi, and P. Krysl. Assumed strain nodally integrated hexahedral finite element formulations for elastoplastic applications. International Journal for Numerical Methods in Engineering, 99(11):844-866, 2014. 
[19] A. Ortiz, M.A. Puso, and N. Sukumar. Maximum-entropy meshfree method for compressible and near-incompressible elasticity. Computer Methods in Applied Mechanics and Engineering, 199:1859-1871, 2010.

[20] M. Arroyo and M. Ortiz. Local maximum-entropy approximation schemes: a seamless bridge between finite elements and meshfree methods. International Journal for Numerical Methods in Engineering, 65(13):2167-2202, 2006.

[21] B. Li, F. Habbal, and M. Ortiz. Optimal transportation meshfree approximation schemes for fluid and plastic flows. International Journal for Numerical Methods in Engineering, 83:1541-1579, 2010.

[22] J.A. Nelder and R. Mead. A simplex method for function minimization. Computer Journal, 7:308-313, 1965.

[23] S. López-Querol, P. Navas, J. Peco, and J. Arias-Trujillo. Changing impermeability boundary conditions to obtain free surfaces in unconfined seepage problems. Canadian Geotechnical Journal, 48:841-845, 2011.

[24] P. Navas and S. López-Querol. Generalized unconfined seepage flow model using displacement based formulation. Engineering Geology, 166:140-141, 2013 .

[25] M. A. Biot. Theory of propagation of elastic waves in a fluid-saturated porous solid. I. Low-Frequency range. Journal of the Acoustical Society of America, 28(2):168-178, 1956.

[26] S. López-Querol. Modelización geomecánica de los procesos de densificación, licuefacción y movilidad cíclica de suelos granulares sometidos a solicitaciones dinámicas (in Spanish). PhD thesis, University of Castilla-La Mancha, Ciudad Real, Spain, 2006.

[27] T.J.R. Hughes and H.M. Hilber. Collocation, dissipation and overshoot for time integration schemes in structural dynamics. Earthquake Engineering and Structural Dynamics, 6:99-117, 1978.

[28] E.L. Wilson, I. Farhoomand, and K.J. Bathe. Non-linear dynamic analysis of complex structures. Earthquake Engineering and Structural Dynamics, 1:241$252,1973$.

[29] J.C. Simo and T.J.R. Hughes. On the variational foundations of assumed strain methods. Journal of Applied Mechanics, 53(1):51-54, 1986.

[30] K. V. Terzaghi. Principles of Soil Mechanics. Engineering News-Record, 95:19$27,1925$. 
[31] P.L. Berry and D. Reid. Introduction to Soil Mechanics. McGraw-Hill, Inc., London, United Kingdom, 1987.

[32] J.A. Fernández Merodo, P. Mira, M. Pastor, and T. Li. GeHoMadrid User Manual. CEDEX, Madrid, 1999. Technical Report.

[33] R.A. Barron. Consolidation of fine-grained soils by drain wells. Transactions of ASCE, 113(1):718-742, 1948.

[34] H. Lamb. On the propagation of tremors over the surface of an elastic solid. Philosophical Transactions A, 203:1-42, 1904.

[35] A.T.F. Chen. Plane strain and axi-symmetric primary consolidation of saturated clays. PhD thesis, Rensselaer Polytechnic Institute, Troy, NY, 1966.

[36] J.H. Prevost. Implicit-explicit schemes for nonlinear consolidation. Computer Methods in Applied Mechanics and Engineering, 39:225-239, 1983.

[37] C. Li, R.I. Borja, and R.A. Regueiro. Dynamics of porous media at finite strain. Computer Methods in Applied Mechanics and Engineering, 193:3837$3870,2004$.

[38] Y. Cai, C. Xu, Z. Zheng, and D. Wu. Vertical vibration analysis of axisymmetric saturated soil. Applied mathematics and mechanics, 27:83-89, 2006. 